\title{
Multi-component droplet heating and evaporation: Numerical simulation versus experimental data
}

\author{
S.S. Sazhin ${ }^{\text {a, }}$, A.E. Elwardany ${ }^{\text {a }}$, P.A. Krutitskii ${ }^{\text {b }}$, V. Deprédurand ${ }^{c}$, G. Castanet $^{\text {c }}$, \\ F. Lemoine ${ }^{c}$, E.M. Sazhina ${ }^{a}$, M.R. Heikal ${ }^{a}$ \\ a Sir Harry Ricardo Laboratories, Centre for Automotive Engineering, School of Computing, Engineering and Mathematics, Faculty of Science and Engineering, \\ University of Brighton, Brighton BN2 4GJ, UK \\ ${ }^{\mathrm{b}}$ Keldysh Institute for Applied Mathematics, Department 4, Miusskaya Sq. 4, Moscow 125047, Russia \\ ${ }^{c}$ LEMTA, Nancy-Université, CNRS UMR 7563, 2 Avenue de la Forêt de Haye, BP 160, 54504 Vandoeuvre-lès-Nancy, France
}

Keywords:

Droplets

Multi-component fuel

Heating

Evaporation

Diffusion equation

Heat conduction

\begin{abstract}
A B S T R A C T
The earlier reported simplified model for multi-component droplet heating and evaporation is generalised to take into account the coupling between droplets and the ambient gas. The effects of interaction between droplets are also considered. The size of the gas volume, where the interaction between droplets and gas needs to be taken into account, is estimated based on the characteristic thermal and mass diffusion scales. The model is applied to the analysis of the experimentally observed heating and evaporation of monodispersed n-decane/3-pentanone mixture droplets at atmospheric pressure. It is pointed out that the effect of coupling leads to noticeably better agreement between the predictions of the model and the experimentally observed average droplet temperatures. In most cases, the observed droplet temperatures lie between the average and central temperatures, predicted by the coupled solution. For the cases reported in this study, the observed time evolution of droplet radii cannot be used for the validation of the model. It is pointed out that the number of terms in the series in the expressions for droplet temperature and species mass fraction can be reduced to just three, with possible errors less than about $0.5 \%$. In this case, the model can be recommended for the implementation into computational fluid dynamics (CFD) codes and used for various engineering applications, including those in internal combustion engines.
\end{abstract}

\section{Introduction}

The problem of modelling multi-component droplet heating and evaporation is a long-standing one, and has been widely discussed in the literature (e.g. [1-8]). A new simplified model for bi-component droplet heating and evaporation was suggested in [9]. This model is based on the analytical solutions for heat conduction and species diffusion equations inside droplets, which are incorporated into a numerical code. The results of calculations were validated against experimental data obtained at the University of Nancy (France).

Although the model, suggested in [9], was recommended for incorporation into computational fluid dynamics (CFD) codes, there are still a number of issues which need to be addressed. The original model of [9], took into account the effect of ambient gas on droplets

\footnotetext{
* Corresponding author. Tel.: +44 1273642 677; fax: +44 1273642309 .

E-mail address: s.sazhin@brighton.ac.uk (S.S. Sazhin).
}

but ignored the effects of droplets on gas. Furthermore, the issue of optimisation of the code was not addressed in [9]. The choice of the number of terms in the analytical solution was based exclusively on the numerical values of the ignored terms, without taking into account the computational cost. These two issues will be addressed in this paper. Also, the model suggested in [9] will be generalised to arbitrary number of species and validated against experimental data different from those used in [9]. The new analytical solution for species was given in [9], without showing the details of the derivation. Since these details are not trivial, they are reproduced in this paper.

As in [9], we will assume that the surface of the droplet remains spherical (see [10-12] for modelling the time evolution of the droplet surface using the Volume of Fluid methodology), and its radius remains constant during the time step (this assumption was relaxed in the most recent papers $[13,14]$ ).

Basic equations and approximations are briefly summarised in Section 2. Our approach to the coupled solution and the numerical algorithm are described in Sections 3 and 4 . Section 5 is focused on 


\begin{tabular}{|c|c|c|c|}
\hline \multicolumn{2}{|c|}{ Nomenclature } & $X$ & molar fraction \\
\hline$A_{M . T}$ & coefficients in Correlations (24) and (25) & Y & mass fraction \\
\hline$B_{M}$ & Spalding mass transfer number & \multirow{2}{*}{\multicolumn{2}{|c|}{ Greek symbols }} \\
\hline$B_{T}$ & Spalding heat transfer number & & \\
\hline c & specific heat capacity & $\alpha$ & parameter defined by Eq. (17) \\
\hline$C_{M . T}$ & coefficients in Correlations (24) and (25) & $\delta$ & film thickness \\
\hline$d$ & distance between droplets & $\gamma$ & activity coefficient \\
\hline$D$ & diffusion coefficient & $\epsilon$ & evaporation rate \\
\hline$f$ & frequency of droplet production & $\varepsilon_{v a}$ & Lennard-Jones energy \\
\hline$F$ & function introduced in Eqs (5)-(6) & $\Theta$ & function introduced in Eq. (50) \\
\hline$G_{M . T}$ & coefficients in Correlations (24) and (25) & $\kappa$ & thermal diffusivity \\
\hline$h$ & convection heat transfer coefficient & $\lambda, \lambda_{n}$ & eigenvalues \\
\hline$h_{0}$ & $-\frac{\alpha}{D_{l}} R_{d}-1$ & $\mu$ & dynamic viscosity \\
\hline$k$ & $\begin{array}{l}D_{l} \\
\text { thermal conductivity }\end{array}$ & $\nu$ & kinematic viscosity \\
\hline$L$ & latent heat of evaporation & $\rho$ & density \\
\hline Le & Lewis number & $\sigma$ & Lennard-Jones length \\
\hline$m$ & mass & $\varphi$ & parameter defined by Eq. (12) \\
\hline M & molar mass & $\chi$ & parameter defined by Eq. (14) \\
\hline$N$ & number of moles or species & $\chi_{Y}$ & parameter defined by Eq. (20) \\
\hline$N_{R}$ & the number of points along the radius inside droplets & $\Omega_{D}$ & function introduced in Eq. (41) \\
\hline $\mathrm{Nu}$ & Nusselt number & & \\
\hline$p$ & pressure & \multicolumn{2}{|c|}{ Subscripts } \\
\hline $\mathrm{Pe}$ & Peclet number & air & air \\
\hline $\operatorname{Pr}$ & Prandtl number & $d$ & droplet \\
\hline$q_{\text {in }}$ & parameter defined by Eq. (74) & $e$ & evaporation \\
\hline$Q_{n}$ & parameter defined by Eq. (69) & eff & effective \\
\hline$R$ & distance from the droplet centre & $g$ & gas \\
\hline$R_{u}$ & universal gas constant & $i$ & species \\
\hline$R_{d}$ & droplet radius & iso & isolated \\
\hline $\operatorname{Re}$ & Reynolds number & $l$ & liquid \\
\hline Sc & Schmidt number & M & mass boundary layer \\
\hline Sh & Sherwood number & $p$ & constant pressure \\
\hline$t$ & time & $r$ & radial \\
\hline$T$ & temperature & $s$ & surface \\
\hline$u$ & variable introduced in Eq. (47) & $T$ & thermal boundary layer \\
\hline$V_{g}$ & interaction volume & $v$ & vapour \\
\hline$v_{n}$ & eigen function & 0 & initial \\
\hline$v_{r}$ & radial velocity & $\infty$ & ambient conditions \\
\hline
\end{tabular}

the experimental setup and input parameters. The results of calculation are compared with experimental data in Section 6. In Section 7, the accuracy of the model predictions versus the CPU efficiency of the code are investigated. The main results of the paper are summarised in Section 8.

\section{Basic equations and approximations}

Most basic equations and approximations used in our analysis are essentially the same as used in [9]. They are briefly summarised below. New equations, not used in [9], are described in more detail.

Droplets are assumed to be perfect spheres, and the process of their heating is described by the following equation $[15,16]$

$\frac{\partial T}{\partial t}=\kappa\left(\frac{\partial^{2} T}{\partial R^{2}}+\frac{2}{R} \frac{\partial T}{\partial R}\right)$,

where $\kappa=k_{l} /\left(c_{l} \rho_{l}\right)$ is the liquid thermal diffusivity. Eq. (1) is to be solved for $t>0$ and $0 \leq R \leq R_{d}$, where $R_{d}$ is the droplet radius, with the following boundary condition (ignoring the effect of evaporation):
$h\left(T_{g}-T_{s}\right)=\left.k_{l} \frac{\partial T}{\partial R}\right|_{R=R_{d}-0}$,

and the initial condition $T(t=0)=T_{d 0}(R)$, where $T_{S}=T_{S}(t)$ is the droplet's surface temperature, $T_{g}=T_{g}(t)$ is the ambient gas temperature, subscript ${ }_{-0}$ indicates that the derivative is taken inside the droplet. The analytical solution to this equation is given and discussed in [9]. This solution is applied at individual timesteps. To take into account the effect of droplet evaporation, gas temperature should be replaced by the so-called effective temperature defined as $T_{\text {eff }}=T_{g}+\rho_{l} L \dot{R}_{d e} / h$, where the value of $\dot{R}_{d e}$ is taken from the previous time step and estimated based on the following equation:

$\dot{R}_{d e}=\frac{\dot{m}_{d}}{4 \pi R_{d}^{2} \rho_{l}}=\frac{-2 \pi R_{d} D_{v} \rho_{\text {total }} B_{\mathrm{M}} S h}{4 \pi R_{d}^{2} \rho_{l}}$,

where $D_{v}$ is the binary diffusion coefficient of vapour in air, $B_{M}$ is the Spalding mass transfer number defined as:

$B_{M}=\frac{\rho_{v s}-\rho_{v \infty}}{\rho_{g s}}=\frac{Y_{v s}-Y_{v \infty}}{1-Y_{v s}}$, 
$Y_{v}=\sum_{i} Y_{v i}$ is the vapour mass fraction, Sh is the Sherwood number. This number and the Nusselt number for an isolated droplet $\left(\mathrm{Sh}_{\text {iso }}\right.$ and $\left.\mathrm{Nu}_{\text {iso }}\right)$ were estimated based on the model suggested in [17] (this model was extended to a moving multicomponent droplet in [18]):

$\mathrm{Sh}_{\text {iso }}=2 \frac{\ln \left(1+B_{M}\right)}{B_{M}}\left(1+\frac{\left(1+\operatorname{Re}_{d} S c_{d}\right)^{1 / 3} \max \left[1, \operatorname{Re}_{d}^{0.077}\right]-1}{2 F\left(B_{M}\right)}\right)$,

$\mathrm{Nu}_{i s o}=2 \frac{\ln \left(1+B_{T}\right)}{B_{T}}\left(1+\frac{\left(1+\operatorname{Re}_{d} \operatorname{Pr}_{d}\right)^{1 / 3} \max \left[1, \operatorname{Re}_{d}^{0.077}\right]-1}{2 F\left(B_{T}\right)}\right)$,

where

$\mathrm{Sc}_{d}=\frac{\nu_{\text {air }}}{D_{v}}$

is the Schmidt number,

$B_{T}=\frac{c_{p v}\left(T_{g}-T_{S}\right)}{L_{\mathrm{eff}}}$

is the Spalding heat transfer number,

$F\left(B_{M, T}\right)=\left(1+B_{M, T}\right) \frac{0.7}{\ln \left(1+B_{M, T}\right)} B_{M, T}$,

$L_{\mathrm{eff}}=L+\frac{Q_{L}}{\dot{m}_{d}}=\sum_{i} \epsilon_{i} L_{i}+\frac{Q_{L}}{\sum_{i} \dot{m}_{i}}$,

$Q_{L}$ is the power spent on droplet heating, $c_{p v}$ is the specific heat capacity of fuel vapour, $\epsilon_{i}=\epsilon_{i}(t)$ are the evaporation rates of species $i, \dot{m}_{i}=\epsilon_{i} \dot{m}_{d}\left(\dot{m}_{d}=\sum_{i} \dot{m}_{i}\right) . B_{T}$ and $B_{M}$ are linked by the following equation [17]:

$B_{T}=\left(1+B_{M}\right)^{\varphi}-1$,

where

$\varphi=\left(\frac{c_{p v}}{c_{p, \mathrm{air}}}\right)\left(\frac{\mathrm{Sh}^{*}}{\mathrm{Nu}^{*}}\right) \frac{1}{\mathrm{Le}}$,

Le $=k_{g} /\left(c_{p, \text { air }} \rho_{\text {total }} D_{v}\right)=\mathrm{Sc} / \operatorname{Pr}$ is the Lewis number,

$\mathrm{Sh}^{*}=2\left(1+\frac{\left(1+\mathrm{Re}_{d} S \mathrm{Sc}_{d}\right)^{1 / 3} \max \left[1, \mathrm{Re}_{d}^{0.077}\right]-1}{2 F\left(B_{M}\right)}\right)$,

$\mathrm{Nu}^{*}=2\left(1+\frac{\left(1+\operatorname{Re}_{d} \operatorname{Pr}_{d}\right)^{1 / 3} \max \left[1, \operatorname{Re}_{d}^{0.077}\right]-1}{2 F\left(B_{T}\right)}\right)$.

When deriving (9) and (10) we assumed that the corrections to Sh${ }^{*}$ and $\mathrm{Nu}^{*}$ due to finite distance between droplets are the same. Remembering that $F\left(B_{M, T}\right)$ is a slowly varying function in the range from 1 to 1.285 [17] and assuming that Le is close to 1 , we can expect that

$\left(\frac{\mathrm{Sh}^{*}}{\mathrm{Nu}^{*}}\right) \approx 1$

Note that for small Re, condition (11) is always satisfied. For large $\mathrm{Re},\left(\mathrm{Sh}^{*} / \mathrm{Nu}^{*}\right) \approx \mathrm{Le}^{1 / 3}$. In this case, even if $\mathrm{Le}=1.5$, the error introduced by Approximation (11) would be less than 15\%. This error is comparable or even less than the errors due to different dependencies of $\mathrm{Sh}^{*}$ and $\mathrm{Nu}^{*}$ on spacing between droplets (cf. Eqs. (24) and (25)). Remembering (11), Eq. (8) is simplified to:

$\varphi=\left(\frac{c_{p v}}{c_{p, \text { air }}}\right) \frac{1}{\text { Le }}$.

The latter expression for $\varphi$ is used in our analysis. This approach allowed us to avoid the iteration procedure to take into account the dependence of $\varphi$ on $B_{M, T}$.

The thermal expansion or contraction of droplets was taken into account as in [9]. The effect of recirculation inside droplet was taken into account based on the effective thermal conductivity (ETC) model [17] in which the liquid thermal conductivity $k_{l}$ is replaced by the effective thermal conductivity

$k_{\mathrm{eff}}=\chi k_{l}$,

where the coefficient $\chi$ is defined as

$\chi=1.86+0.86 \tanh \left[2.225 \log _{10}\left(\mathrm{Pe}_{d(l)} / 30\right)\right]$,

$\mathrm{Pe}_{d(l)}=\mathrm{Re}_{d(l)} \operatorname{Pr}_{l}$ is the droplet Peclet number, in which the liquid transport properties and the relative velocities between droplets and gas were used.

All transport coefficients for the mixtures and individual substances were estimated as discussed in Appendix 1. Note that our analysis is focused on the evaporation only; the analysis of condensation is beyond the scope of this paper.

Equations for mass fractions of liquid species $Y_{l i} \equiv Y_{l i}(t, R)$ inside droplets were used in the following form:

$\frac{\partial Y_{l i}}{\partial t}=D_{l}\left(\frac{\partial^{2} Y_{l i}}{\partial R^{2}}+\frac{2}{R} \frac{\partial Y_{l i}}{\partial R}\right)$,

where $i>1, D_{l}$ is the liquid mass diffusivity. Eq. (15) was solved with the following boundary condition [1]:

$\alpha\left(\epsilon_{i}-Y_{\text {lis }}\right)=-\left.D_{l} \frac{\partial Y_{l i}}{\partial R}\right|_{R=R_{d}-0}$,

and the initial condition $Y_{l i}(t=0)=Y_{l i 0}(R)$, where $Y_{\text {lis }}=Y_{\text {lis }}(t)$ are liquid components' mass fractions at the droplet's surface,

$\alpha=\frac{\left|\dot{m}_{d}\right|}{4 \pi \rho_{l} R_{d}^{2}}=\left|\dot{R}_{d e}\right|$

Assuming that species concentrations in the ambient gas are equal to zero $\left(Y_{v \infty}=0\right)$, the values of $\epsilon_{i}$ can be found from the following relation [9]:

$\epsilon_{i}=\frac{Y_{v i s}}{\sum_{i} Y_{v i s}}$

where the subscript ${ }_{v}$ indicates the vapour phase. We assume that $\epsilon_{i}$ are still defined by Eq. (18) even in the case when $Y_{v \infty} \neq 0$, which is expected in the case of a coupled solution. The analytical solution of Eq. (15) is given in Appendix 2. This solution is generalised to the case of moving droplets with the help of the effective diffusivity model $[1,9]$ in which $D_{l}$ in Eq. (15) is replaced with the so-called effective diffusivity $D_{\text {eff }}[37]$ :

$D_{\text {eff }}=\chi_{Y} D_{l}$,

where

$\chi_{Y}=1.86+0.86 \tanh \left[2.225 \log _{10}\left(\operatorname{Re}_{d(l)} \mathrm{Sc}_{l} / 30\right)\right]$ 
To calculate the species mass evaporation rate $\dot{m}_{d i}$ and the values of the evaporation rate of species $\epsilon_{i}$ based on Eq. (18) we need to calculate first the values of $Y_{v i s}$. The latter depend on the partial pressure of species $i$ in the vapour state in the immediate vicinity of the droplet surface [19]:

$p_{v i}=X_{l i s} \gamma_{i} p_{v i}^{*}$

where $X_{\text {lis }}$ is the molar fraction of the $i$ th species in the liquid near the droplet surface, $p_{v i}^{*}$ is the partial vapour pressure of the $i$ th species in the case when $X_{l i s}=1, \gamma_{i}$ is the activity coefficient. In the limit when $\gamma_{i}=1$ (ideal mixture), Eq. (21) describes the Raoult law. This approximation will be used in our analysis. As shown in [9] for the acetone/ethanol mixtures, this can lead to errors in predicted temperatures not more than about $2 \mathrm{~K}$, which is comparable with experimental uncertainties.

The corrections to $\mathrm{Nu}_{\text {iso }}$ and $\mathrm{Sh}_{\text {iso }}$ due to the finite distance between droplets were calculated in [9] based on the empirical correlation which was the function of the distance parameter (the ratio of the distance between droplets and their diameters) (see Eq. (28) in [9]). However, as shown in [20], this correlation does not always work, mainly due to the fact that it does not account for fuel volatility which influences the interaction between droplets. In [21], the ratios $\mathrm{Sh} / \mathrm{Sh}_{\text {iso }}$ and $\mathrm{Nu} / \mathrm{Nu}_{\text {iso }}$ were estimated based on experimental measurements for mono-component droplets. This approach is believed to be not convenient in practical applications.

As shown in [20], the above ratios are controlled not only by the distance parameter but also by the nature of the fuel. Following [20], we introduce the dimensionless time $t^{*}$ defined as:

$t^{*}=\frac{\delta f}{v_{r}}$

where $f$ is the frequency of droplet production (in $\mathrm{Hz}$ ) (setup for each particular experiment and directly linked with the distance parameter), $\delta$ is the film thickness, as introduced in [17], which is different for mass and thermal boundary layers $\left(\delta_{M}\right.$ and $\left.\delta_{T}\right), v_{r}$ is the radial velocity of the vapour released at the droplet surface estimated as

$v_{r}=\frac{\dot{m}_{d}}{4 \pi \rho_{v} R_{d}^{2}}$,

$\rho_{v}$ is the density of vapour. Thus parameter $t^{*}$ takes into account the contributions of both the distance parameter (via $f$ ) and the volatility of fuel (via $v_{r}$ ).

The values for $\delta_{M}$ and $\delta_{T}$ for mono-component droplets were estimated based on the model suggested in [17] (see also [1]). The definitions of these thicknesses and $v_{r}$ were generalised to the case of multi-component droplets, via introduction of the average density and mass averaged values of transport coefficients (cf. [9]). Having introduced the concept of $t^{*}$, Deprédurand et al. [20] suggested the following correlations for $\mathrm{Nu} / \mathrm{Nu}_{\mathrm{iso}}$ for acetone, ethanol, 3-pentanone, n-decane, n-dodecane and n-heptane, and $\mathrm{Sh} / \mathrm{Sh}_{\text {iso }}$ for ethanol, 3-pentanone and n-heptane $\left(\mathrm{Sh} / \mathrm{Sh}_{\text {iso }}\right.$ for acetone was approximated by the same correlation as for $\mathrm{Nu} / \mathrm{Nu}_{\mathrm{iso}}$ ):

$\frac{\mathrm{Nu}}{\mathrm{Nu}_{\mathrm{iso}}}=\frac{1-A_{T}}{\left(G_{T} t^{*}+1\right)^{C_{T}}}+A_{T}$,
$\frac{\mathrm{Sh}}{\mathrm{Sh}_{\mathrm{iso}}}=\frac{1-A_{M}}{\left(G_{M} t^{*}+1\right)^{C_{M}}}+A_{M}$,

where the coefficients used in these equations are given in Table 1. Equations (24) and (25) describe the effects of spacing between droplets on the values of $\mathrm{Nu}$ and $\mathrm{Sh}$.

Although Correlations (24) and (25) were derived for a limited number of substances, we assume that they can be used for a wider range of substances and their mixtures. They will be applied for the analysis of droplets of the mixture of n-decane and 3-pentanone.

\section{The coupled solution}

The model, described in the previous section, could be generalised to take into account the effects of droplets on gas and then incorporated into any computational fluid dynamics (CFD) code (e.g. KIVA [22]). This analysis, however, is beyond the scope of this paper. Instead, a simplified model, capturing the essential features of coupling between droplets and ambient gas will be described with a view to a specific application to the analysis of the experiments described in $[5,20,23]$. In these experiments, ambient gas pressure remained the same and equal to the atmospheric pressure. This model will be eventually applied to the analysis of the experiments similar to those described in the abovementioned papers.

Let us assume that the droplet exchanges heat and mass with a certain volume $V_{g}$, surrounding the droplet. Following [24], we call it the region of influence. The shape of this region can be either spherical, in the case of isolated droplets, or cylindrical, in the case of droplet streams, considered in $[5,20,21,23]$. In both cases, the cross-section of this region in arbitrary direction for the spherical region and the cross-section perpendicular to the axis of the cylinder can be schematically presented as shown in Fig. 1. For the spherical and cylindrical regions, their volumes can be estimated based on the following equations

$V_{g}=\frac{4}{3} \pi\left(R_{g}^{3}-R_{d}^{3}\right)$

and

$V_{g}=\pi R_{g}^{2} d-\frac{4}{3} \pi R_{d}^{3}$

respectively, where $d$ is the distance between droplets. Since the cylindrical region is more physical for the cases considered in our paper, the focus of our analysis will be mainly on this particular region. The ratio $r=V_{g} / V_{d}$, where $V_{d}=4 / 3 \pi R_{d}^{3}$, was fixed during calculations but the values of $R_{g}$ were recalculated at each time step depending on the volume of the droplet. Since the changes of $V_{d}$ were small for the cases to be considered, the values of $R_{g}$ remained close to their initial values. The choice of the initial values of $R_{g}$ will be discussed in the next section.

\subsection{Size of the region of influence}

In CFD codes the results of calculations should not depend on the cell sizes for sufficiently small cells, since the ordinary differential equations in individual cells are solved alongside partial

Table 1

The numerical values of parameters in Eqns. (24) and (25).

\begin{tabular}{|c|c|c|c|c|c|c|}
\hline Parameter & $A_{T}$ & $G_{T}$ & $C_{T}$ & $A_{M}$ & $G_{M}$ & $C_{M}$ \\
\hline Value & 0.155335 & 1.416767 & 0.535026 & 0.155335 & $6.503406 \times 10^{-7}$ & 54302.36 \\
\hline
\end{tabular}




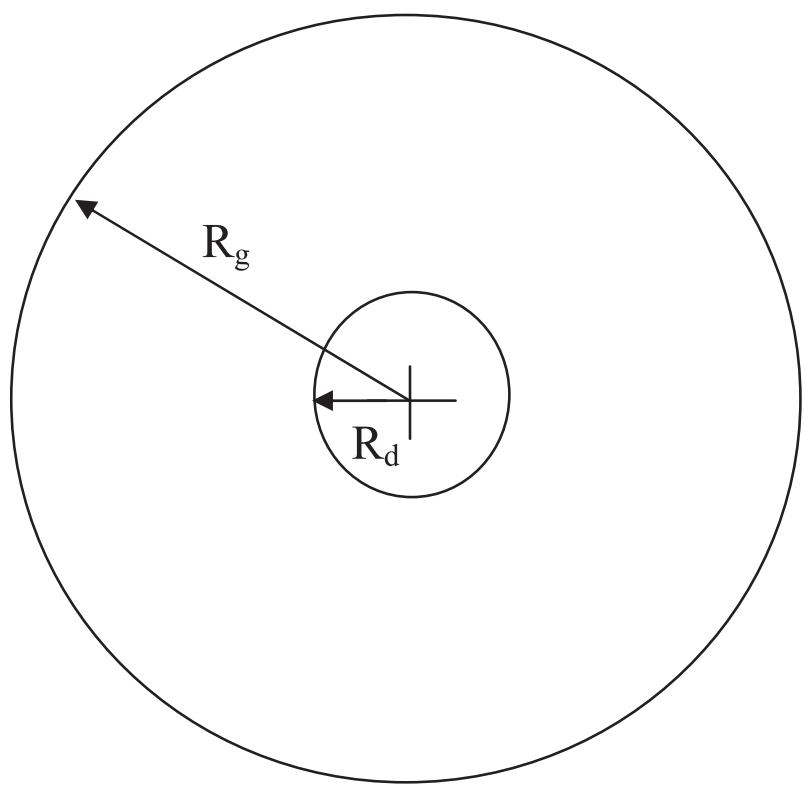

Fig. 1. Schematic diagram for the spherical region of influence or the plane perpendicular to the cylinder axis for the cylindrical region of influence.

differential equations describing mass, momentum and energy conservation between cells. Although this is true in the case of the Eulerian approach, the results usually start depending on the grid size for sufficiently small cells in the case of the Lagrangian/Eulerian approach widely used for spray simulation. This problem was extensively discussed in [24], where the concept of the region of influence was first introduced. The main idea of this concept is to allow droplets to exchange mass, momentum and energy not only with gas in a cell, in which a droplet is located, but with gas in a wider à priori determined region (region of influence). Essentially the same idea is used in our approach. The size of this region is considered as a free parameter, within a certain range, which can be adjusted to get the best fit with experimental data. In the case of very large $V_{g}$, the coupled solution reduces to the one-way solution. For small $V_{g}$, we can obtain an unphysical solution, since in this case the interaction of gas in $V_{g}$ and the ambient gas, ignored in our analysis, can play the dominant role.

We appreciate that this approach does not allow us to perform a quantitative predictive analysis of mass, momentum and energy exchange between individual droplets and gas, but it does enable us to perform a simple qualitative analysis of the effect of this exchange on time evolution of droplet temperature and radius. As shown later, it allows us to explain some discrepancies between experimental measurements and the predictions of the one-way model. Note that this model will be applied to the analysis of experiments similar to those described in [5,20,21], where the droplets' velocities were determined from the experiments and not calculated. Hence the momentum transfer between the region of influence and the droplets is not considered in our model. In this case, we can approximate $R_{g}$ as

$R_{g}=R_{d}+\sqrt{\kappa_{D} t_{D}}$

where $t_{D}$ is the characteristic duration of the process,

$\kappa_{D}=\max \left(\frac{k_{g}}{c_{p g} \rho_{g}}, D_{v}\right)$,

where the first term in the latter equation describes the heat diffusivity in volume $V_{g}$, while the second term describes diffusion coefficient of the fuel vapour in the gas inside the region of influence. Equations (28) and (29) allow us to assume that this region of influence is large enough to enable us to ignore heat exchange between it and the ambient gas, and escape of fuel vapour from this volume to the ambient gas.

Let us first focus on the mass transfer process.

\subsection{Mass balance}

The total number of moles of gas inside volume $V_{g}$ can be obtained from the ideal gas law

$N_{\text {total }}=\frac{p_{\mathrm{atm}} V_{g}}{R_{u} T}$,

where $R_{u}$ is the universal gas constant. Initially, there is no vapour in $V_{g}$ and $N_{\text {total }}=N_{\text {air }}$ at $T=T_{0}$. Once the evaporation process has started then a certain number of moles of vapour $N_{v}=\sum_{i=1}^{N} N_{v i}$, where $N$ is the total number of species, penetrates into volume $V_{g}$. Simultaneously the temperature changes from the initial temperature $T_{0}$ to $T_{1}$. The number of moles of air in this case reduces (or increases if $T_{1}$ is sufficiently lower than $T_{0}$ ) to:

$N_{\mathrm{air}}=\frac{p_{\mathrm{atm}} V_{g}}{R_{u} T_{1}}-\sum_{i=1}^{N} N_{v i}$.

This allows us to calculate the mass fraction of vapour inside the cell as

$Y_{v(\text { cell })}=\frac{\sum_{i=1}^{N} M_{v i} N_{v i}}{\sum_{i=1}^{N} M_{v i} N_{v i}+M_{\mathrm{air}} N_{\mathrm{air}}}$,

where $M_{\mathrm{air}}$ is the molar mass of air, $M_{v i}$ are molar masses of vapour species. We assume that $Y_{v \infty}=Y_{v(\text { cell })}$. In the one-way solution described in [9] it was assumed that $Y_{v \infty}=0$. When deriving Eqs. (31) and (32) we used our assumption that the region of influence is large enough to prohibit the fuel vapour from escaping from volume $V_{g}$. For sufficiently small timesteps, we can assume that $T_{1}=T_{0}$ in Eq. (31) and updated at the next time step.

The calculation continues until $Y_{v(\text { cell })}=Y_{v s}$. Once this happens, the droplet stops evaporating $\left(B_{M}=0\right)$. Apart from $Y_{v(\text { cell })}$, Eqs. (31) and (32) are used for calculation of physical properties of the mixture of vapour and air.

\subsection{Heat balance}

Assuming that gas temperature inside volume $V_{g}$ is homogeneous, the time evolution of this temperature can be described by the equation:

$\frac{\mathrm{d}\left(m_{g} c_{p g} T_{g}\right)}{\mathrm{d} t}=-2 \pi k_{g} \mathrm{Nu}_{d} R_{d}\left(T_{g}-T_{S}\right)$

where $m_{g}$ is the total mass of gas (mixture of air and vapour) in volume $V_{g}, \mathrm{Nu}_{d}$ is the Nusselt number for evaporating droplets (taking into account the heating/cooling of the vapour).

Eq. (33) was solved at each time step. The value of $m_{g}$ in this equation is the mass of the mixture of air and fuel vapour as calculated at the previous time step. The values of $k_{g}$ and $c_{p g}$ were calculated for the mixture of air and fuel vapour. The physical meaning of Eq. (33) is obvious: this is the mathematical expression of the statement that the energy lost by the gas is spent on droplet heating and evaporation.

Note that in our approach to the coupled solution we implicitly took into account the transient nature of the process of droplet heating and evaporation. At the same time, the expressions for $\mathrm{Nu}$ 
and Sh, discussed in Section 2, were derived under the assumption that the process is quasi-steady (heat and mass flow rate through each sphere surrounding the droplet remains the same). Transient effects on $\mathrm{Nu}$ have been considered in a number of papers, including [25-27]. As shown in [25], the quasi-steady approximation is valid at times much greater than $t_{c} \equiv R_{d}^{2} c_{p} \rho_{g} /\left(\pi k_{g}\right)$. For droplets with radii about $60 \mu \mathrm{m}$ and typical parameters for the experiment considered in our paper, $t_{c} \approx 0.25 \mu \mathrm{s}$. The time scales of the processes under consideration are much greater than $0.25 \mu \mathrm{s}$. We anticipate that the quasi-steady approximation for Sh is valid after a certain $\tilde{t}_{c}$ which is of the same order of magnitude as the abovementioned $t_{c}$. The approach which we use is consistent with widely used practice in Computational Fluid Dynamics (CDF) codes $[1,24]$. For sufficiently large computational cells, only a small fraction of mass and heat exchanged between gas and droplets is lost in the ambient gas.

\section{Numerical algorithm}

The numerical scheme outlined below is specifically focused on the application of the model for interpretation of the experimental results reported in $[5,20,23]$. In the experiments reported in these papers ambient pressure was constant. The time evolution of droplet velocities was directly measured and these were used as input parameters of the model at each time step. In contrast to [9], the effects of droplets on the gas phase were taken into account (coupled solution).

These are the main steps of the numerical algorithm:

1. Assume the initial distribution of temperature and mass fractions of species inside the droplet or use the distributions obtained at the previous time step (in our case the initial distributions of both were assumed homogeneous). Recalculate the mass fractions of species into molar fractions of species.

2. Calculate the values of liquid thermal conductivity and effective thermal conductivity of the droplet. Use the values of the droplet velocities from the experimental data.

3. Calculate species partial pressures and molar fractions in the gas phase from the Raoult law.

4. Calculate maximum of the thermal and mass diffusivities; calculate $R_{g}$ according Eq. (28) with $t_{D}=12 \mathrm{~ms}$ (typical transit time of droplets).

5. Calculate the interaction volume based on Eq. (26) for the spherical volume and Eq. (27) for the cylindrical volume where $d=C \times 2 \times R_{d}, C$ is the distance parameter.

6. Calculate the concentration of vapour of all species in the region of influence and the value of the Spalding mass transfer number.

7. Calculate the values of heat capacity, diffusivity of the mixture of vapour species in the air and species evaporation rates $\left(\epsilon_{i}\right)$.

8. Calculate the values of Nusselt and Sherwood numbers for isolated droplets.

9. Calculate the values of Nusselt and Sherwood numbers for droplets, taking into account the interaction between them (Eqs. (24) and (25)).

10. Calculate the total change of mass of the evaporating droplet during the time step $\Delta t$, taking into account the interaction between droplets and the change in liquid density.

11. Calculate the rate of change of droplet radius.

12. Calculate the distribution of temperature inside the droplet.

13. Calculate the distribution of species inside the droplet.

14. Recalculate the droplet's radius at the end of the time step $\Delta t$. In our analysis the droplets never fully evaporated, but the program was designed to deal with the case when the complete evaporation takes place, if necessary. If this radius is negative then the time step is reduced and the calculations are repeated. If the ratio of this radius to the initial radius is less than an a priori chosen small number $\epsilon_{s}=10^{-6}$, then the remaining part of the droplet is assumed to be evaporated with all liquid species transferred into the gas phase with the corresponding decrease in gas temperature. If this ratio is greater than $10^{-6}$ then go to the next step.

15. Recalculate the distributions of temperature and species for the new radius (e.g. $T(R)=T\left(R R_{d 2} / R_{d 1}\right)=T(\tilde{R})$, where $R_{d 1,2}$ are droplet radii at the beginning and the end of the time step, $\tilde{R}$ is the new $R$ used at the second time step, $T$ are the values of temperature at the end of the time step).

16. Return to Step 1 and repeat the calculations for the next time step.

\section{Experimental setup and input parameters}

The experimental setup used for validation of the model is the same as described in $[20,21]$. Monodispersed and periodically spaced droplets were generated by the break-up of a liquid jet via Rayleigh-type instability at the injector outlet. A piezoelectric ceramic inserted into the injector body triggered the disintegration of the liquid jet by vibrations at certain frequencies. The fuel temperature was regulated in the injector body and a thermocouple (located near the outlet of the injector) was used to verify that the set temperature was maintained the same during the experiment. The droplets were injected into a hot air enclosure equipped with several optical windows for measurements. Air within the enclosure was preheated up to $400{ }^{\circ} \mathrm{C}$. The walls of the enclosure were made of thermally isolating materials, and an electric wire was inserted into the interior of the walls. The electric current was adjusted so that the Joule heating could compensate thermal losses. A constant air flow was maintained to avoid the risk of progressive saturation of vapour in the enclosure. An almost uniform air velocity in the cross-section of the enclosure was achieved by introducing a layer of metal foam at the air inlet. Air velocity of about $0.3 \mathrm{~m} / \mathrm{s}$ turned out to be sufficient to deplete vapour from the enclosure. The relatively large radius of the enclosure $(0.05 \mathrm{~m})$ and small diffusion length did not allow vapour to diffuse to the walls before it was removed through the holes drilled at the top of the enclosure.

Downstream distance from the injector was converted into time with the help of the space evolution of the droplet velocity measured using the Laser Doppler Anemometry (LDA) technique. Droplet temperatures were measured using the laser induced fluorescence technique described in $[28,20]$. The emissions from two different spectral bands with different temperature sensitivities were utilized. The ratio of the intensities of these two bands allowed us to eliminate the effects of parameters that are unknown or difficult to control, such as the variations in laser intensity, tracer concentration or measurement volume during the acquisitions. At the same time this ratio depends on the temperature, making possible its measurement. Although fluorescent tracers like Rhodamine B or Kitton red are well known for the temperature sensitivity of their fluorescent signal and their high quantum yield, the fluorescence spectrum of these molecules can vary significantly with the composition of the liquid mixture in which they are dissolved. To account for this effect, a special method has been developed and described in [29]. This method is based on the inclusion of a third spectral band. However, in the case of the n-decane/3-pentanone mixture, considered in this paper, we found a new fluorescent tracer, Pyrromethene 597-C8, for which the fluorescent ratio is almost independent on the volume fraction of 3-pentanone up to about $15 \%$ with only a negligible effect to the sensitivity of the fluorescent ratio on the temperature [30]. 
Measurements, using this tracer were therefore performed with only two bands. The probe volume (intersection between the laser beams and the detection field of view) was about $150 \times 150 \times 1200 \mu \mathrm{m}^{3}$. It was larger than the droplet in order to provide a global excitation of the whole droplet volume. The signal was averaged over the total time of droplet transit in the probe volume.

In the presence of thermal gradients inside droplets, the temperature obtained, based on this signal, could differ from the volume-averaged droplet temperature. This is related to the fact that the laser intensity is not distributed uniformly inside the droplet, mainly due to light refraction at the droplet surface and the Gaussian profile of intensity in the laser beams. Also, the receiving optics collect the fluorescent signal from the droplet interior with a spatial distortion. These effects could be taken into account with the help of an efficient ray tracing code, based on the geometric optics approximation [31]. This analysis, however, is beyond the scope of this paper. We would just mention that the 2cLIF measurements allow us to estimate the volume-averaged droplet temperature with systematically more weighting in the zones near the centre of the droplet rather than at its edge. We cannot quantify this effect at this stage and we assume that the measured temperatures are close to the droplet average temperatures.

Moreover, the forward scattering interferometry (FSI) was used to determine the droplet diameter with a good accuracy [32]. In the case of a droplet having diameter of $120 \mu \mathrm{m}$, the uncertainty of the diameter measurement using this method was estimated to be about $\pm 1.2 \mu \mathrm{m}$ [33].

Three sets of experimental data referring to the mixtures of decane and 3-pentanone will be used in our analysis. These are $95 \%$ decane - 5\% 3-pentanone, 90\% decane - 10\% 3-pentanone and 85\% decane $-15 \% 3$-pentanone mixture droplets, which will be referred to as Cases 1, 2 and 3 respectively. Droplet initial diameters, ambient temperatures, distance parameters and injection frequencies for these cases are presented in Table 2 . The experimentally observed time evolutions of droplet average temperatures, velocities and diameters are presented in Table 3.

\section{Results}

A crucial parameter which needs to be considered in the coupled solution is the volume of gas where the interaction between droplets and gas is taken into account, or even more important the ratio of this volume and the volume of the droplet $r \equiv V_{g} / V_{d}$. For spherical and cylindrical cells these volumes are linked with parameter $R_{g}$ via Eqs. (26) and (27) respectively. A crude estimate of this parameter is given by Eq. (28). Taking $\tau_{D}$ in this equation equal to $12 \mathrm{~ms}$ (characteristic transit time of droplets), $R_{d}=61.35 \mu \mathrm{m}$ (see Table 2 ), $d=463.806 \mu \mathrm{m}$ (based on the distance parameter equal to 3.78 (see Table 2)), and estimating $\kappa_{D}$ as $3.61 \times 10^{-5} \mathrm{~m}^{2} / \mathrm{s}$, we obtain $R_{g} \approx 7.2004 \times 10^{-4} \mathrm{~m} \approx 720 \mu \mathrm{m}$. This gives us $r \approx 1615$ for the spherical cell, and $r \approx 780$ for the cylindrical one. The latter is more appropriate for the experimental

Table 2

Droplet initial diameters, ambient temperatures, distance parameters and injection frequencies for the 95\% decane - 5\% 3-pentanone mixture droplets (Case 1), 90\% decane - 10\% 3-pentanone mixture droplets (Case 2) and 85\% decane - 15\% 3pentanone mixture droplets (Case 3 ).

\begin{tabular}{llll}
\hline Parameter & Case 1 & Case 2 & Case 3 \\
\hline Initial diameter & $122.7 \mu \mathrm{m}$ & $126.1 \mu \mathrm{m}$ & $127.7 \mu \mathrm{m}$ \\
Ambient temperature & $370{ }^{\circ} \mathrm{C}$ & $374{ }^{\circ} \mathrm{C}$ & $374{ }^{\circ} \mathrm{C}$ \\
Distance parameter & 3.78 & 3.84 & 3.8 \\
Injection frequency & $20600 \mathrm{~Hz}$ & $20600 \mathrm{~Hz}$ & $20500 \mathrm{~Hz}$ \\
\hline
\end{tabular}

Table 3

The experimentally observed time evolutions of droplet average temperatures velocities and diameters for the $95 \%$ decane - 5\% 3-pentanone mixture droplets (Case 1), 90\% decane - 10\% 3-pentanone mixture droplets (Case 2 ) and 85\% decane $15 \%$ 3-pentanone mixture droplets (Case 3). The contribution of gas velocity is ignored in the estimates of Sherwood and Nusselt numbers as in [9]. Time was measured from the moment of injection.

\begin{tabular}{lllll}
\hline Case & Time $(\mathrm{ms})$ & $\begin{array}{l}\text { Average } \\
\text { Temperature }\left({ }^{\circ} \mathrm{C}\right)\end{array}$ & Velocity $(\mathrm{m} / \mathrm{s})$ & Diameter $(\mu \mathrm{m})$ \\
\hline 1 & 4.784 & 28.66 & 7.77 & 122.7 \\
1 & 6.105 & 30.45 & 7.33 & 122.7 \\
1 & 7.476 & 32 & 7.22 & 122.5 \\
1 & 8.897 & 33.9 & 6.96 & 122.7 \\
1 & 10.368 & 36.8 & 6.75 & 123.1 \\
1 & 11.889 & 40.1 & 6.44 & 123.3 \\
2 & 3.86 & 25.6 & 9.34 & 126.1 \\
2 & 4.9 & 26.67 & 9.04 & 125.7 \\
2 & 5.97 & 28.17 & 8.6 & 125.6 \\
2 & 7.07 & 29.9 & 8.25 & 125.5 \\
2 & 8.2 & 31.88 & 8.04 & 125.4 \\
2 & 9.36 & 34.74 & 7.86 & 125.5 \\
3 & 3.86 & 26.3 & 9.2 & 127.7 \\
3 & 4.9 & 27.99 & 8.92 & 127.6 \\
3 & 5.97 & 29.2 & 8.67 & 127.5 \\
3 & 7.07 & 30.83 & 8.38 & 127.2 \\
3 & 8.2 & 32.26 & 8.04 & 127.1 \\
3 & 9.36 & 34.7 & 7.91 & 126.6 \\
3 & 9.36 & 36.2 & 7.69 & 126.3 \\
\hline
\end{tabular}

results considered in this paper. Remembering that this estimate was made for the maximal $\tau_{D}$, and is rather crude by its nature, we can take the actual value of this parameter a bit less than 780 in the coupled solution, but not too small to affect the assumptions of the model.

The calculations were performed for $r \equiv V_{g} / V_{d}=600$ (coupled solution) and $r=5 \times 10^{4}$ (it was shown that for this $r$ the results predicted by the coupled solution are indistinguishable from the results predicted by the one-way solution, described in [9]). Twenty terms in the series in the analytical solution for temperature and species were used in the calculations (the sensitivity of the results with respect to the choice of the number of terms will be investigated in Section 7). As in [9], in both cases the temperatures at the centre of the droplets, the surface of the droplets and the average droplet temperatures were calculated. The values of ambient gas temperature and droplet velocities were the input parameters of the model. Note that although the ambient gas temperature remained constant during each experiment, in the case of the coupled solution gas temperature inside the volume $V_{g}$ was allowed to change with time. This temperature plays the same role as the ambient temperature in the case of the one-way solution. As in [9] the calculations started at the moment when droplets were first observed. The initial droplet temperatures were assumed equal to the measured temperatures of the first observed droplets. As in [9], it is assumed that initially there is no temperature gradient inside droplets.

The time step in the calculations was taken equal to $10^{-5} \mathrm{~s}$, and the number of points along the radius inside droplets, where the temperatures were stored, was taken equal to 2000. The latter number controls the accuracy of the calculation of the spatial derivatives of the temperature and species mass fractions, used in boundary conditions for $T$ and $Y_{l i}$ (see Eqs. (2) and (16)). The reason for this choice of the time step and the number of points will be discussed later in Section 7.

The plots of time evolutions of the temperatures at the centre of the droplets, the surface of the droplets and the average droplet temperatures, predicted by the coupled and one-way solutions for the 95\% decane - 5\% 3-pentanone mixture droplets (Case 1), are shown in Fig. 2. As follows from this figure, all three temperatures 


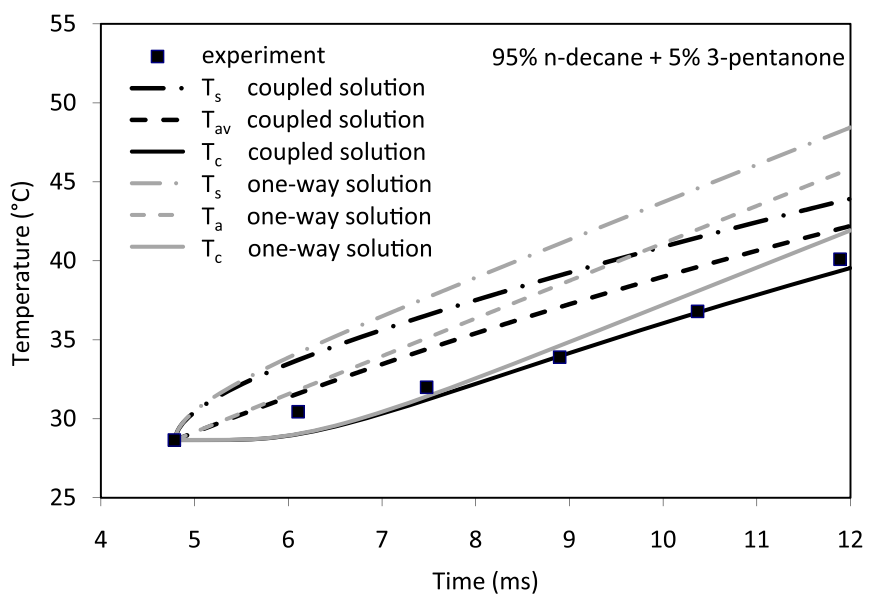

Fig. 2. The time evolution of the droplet surface, average and central temperatures $\left(T_{S}\right.$, $T_{a v}$ and $T_{c}$ ), as predicted by the coupled and one-way solutions, and experimentally observed temperatures for the 95\% n-decane 5\% 3-pentanone mixture droplets with initial diameters $122.7 \mu \mathrm{m}$ and homogeneous temperature $28.66{ }^{\circ} \mathrm{C}$ injected into an ambient gas at constant temperature equal to $370{ }^{\circ} \mathrm{C}$ (see Tables 2 and 3). Zero time corresponds to the start of injection.

under consideration are well separated, as in the cases, considered in [9]. Also, the coupled solutions show visibly slower rates of droplet heating, compared with the predictions of the one-way solution. All experimental plots lie between the average droplet temperature and the temperature at the centre of the droplet predicted by the coupled solution.

The same plots as in Fig. 2 but for the 90\% decane - 10\% 3-pentanone mixture droplets (Case 2) and the $85 \%$ decane - $15 \%$ 3-pentanone mixture droplets (Case 3 ) are shown in Figs. 3 and 4 respectively. The trends of all curves in these figures are rather similar to those shown in Fig. 2. In both cases experimental plots lie between the average droplet temperature and the temperature at the centre of the droplet predicted by the coupled solution, as in the case shown in Fig. 2, except three experimental points in Fig. 4. Even in the latter case, however, the deviation between the predicted temperatures at the centre of the droplet and the ones obtained experimentally is well within the experimental error. In all three cases, the one-way solution predicts unrealistically high average droplet temperatures, well above those observed experimentally. Hence, the effects of coupling need to be taken into account for the accurate prediction droplet temperatures.

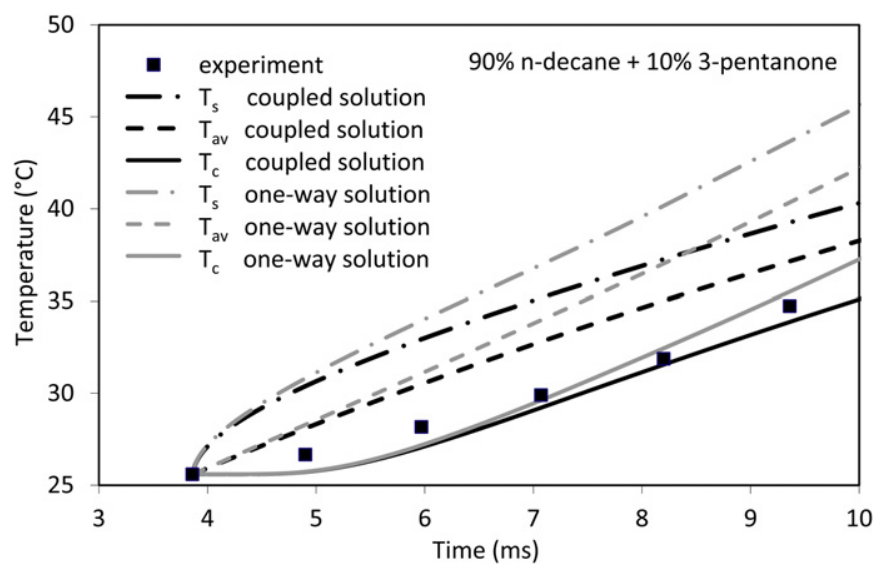

Fig. 3. The same as Fig. 2 but for the $90 \%$ n-decane 10\% 3-pentanone mixture droplets with initial diameters $126.1 \mu \mathrm{m}$, homogeneous temperature $25.6{ }^{\circ} \mathrm{C}$ injected into an ambient gas at constant temperature equal to $374{ }^{\circ} \mathrm{C}$ (see Tables 2 and 3).

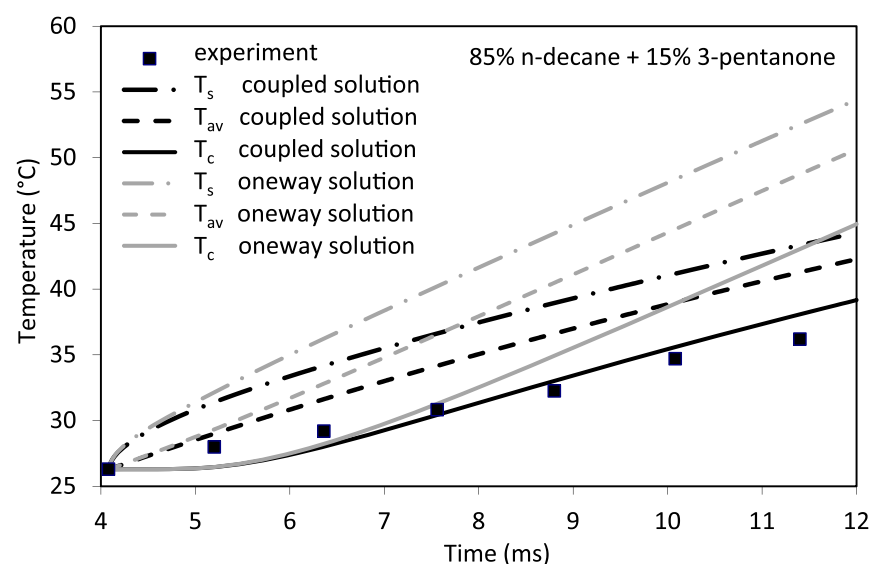

Fig. 4. The same as Figs. 2 and 3 but for the $85 \%$ n-decane $15 \%$ 3-pentanone mixture droplets with initial diameters $127.7 \mu \mathrm{m}$, homogeneous temperature $26.3{ }^{\circ} \mathrm{C}$ injected into an ambient gas at constant temperature equal to $374{ }^{\circ} \mathrm{C}$ (see Tables 2 and 3 ).

The plots of time evolutions of the gas temperatures in the region of influence for all three cases, as predicted by the coupled solution, are shown in Fig. 5. In the case of the one-way solution, this temperature remained constant. As can be seen from Fig. 5, the drop in the gas temperature during droplet heating could reach up to about $70^{\circ} \mathrm{C}$ and this leads to a visible reduction of the rate of increase of droplets temperatures predicted by the coupled solution. This drop in gas temperature is expected to produce much stronger effect due to the coupled solution, compared with the addition of fuel vapour to the region of influence.

The plots, similar to those shown in Figs. 2-4, but for droplet radii, are shown in Figs. 6-8. As one can see from these figures, neither coupled nor one-way solutions can predict the observed trends in the time evolution of droplet radii. At the same time, one can see from these figures that the deviation between the predicted and observed droplet radii is less than about $0.5 \mu \mathrm{m}$ in most cases (less than 1\%), which is within the margins of experimental errors. Hence, the deviations between the predicted and observed droplet radii cannot undermine the validity of the model.

Finally we present four sets of plots, which cannot be validated against experimental data at the moment, but show the underlying physics of the processes involved. The temperature distribution inside the 95\% decane - 5\% 3-pentanone mixture droplets (Case 1) at three moments of time, as predicted by the coupled and one-way

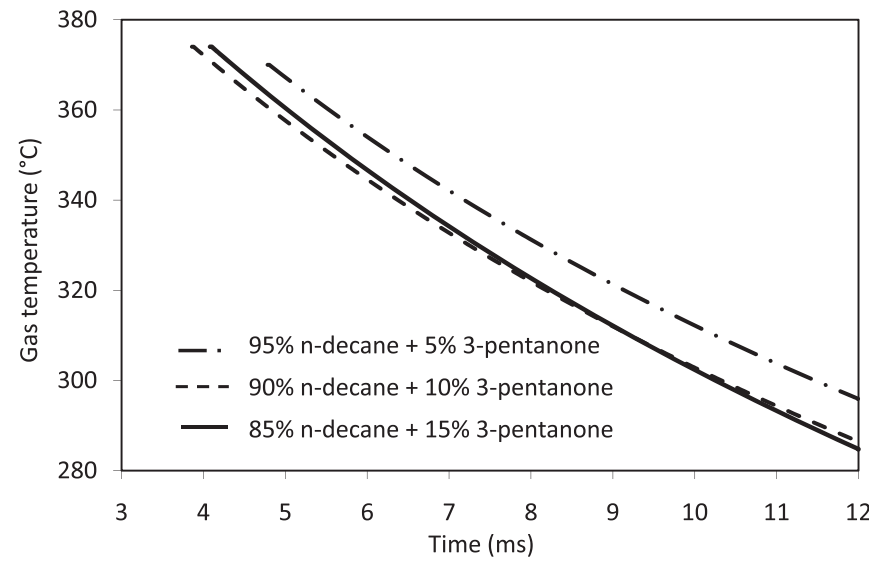

Fig. 5. The time evolution of gas temperature in the region of influence for three mixtures of n-decane and 3-pentanone. The droplets and the ambient gas parameters are the same as used in Figs. 2-4. 


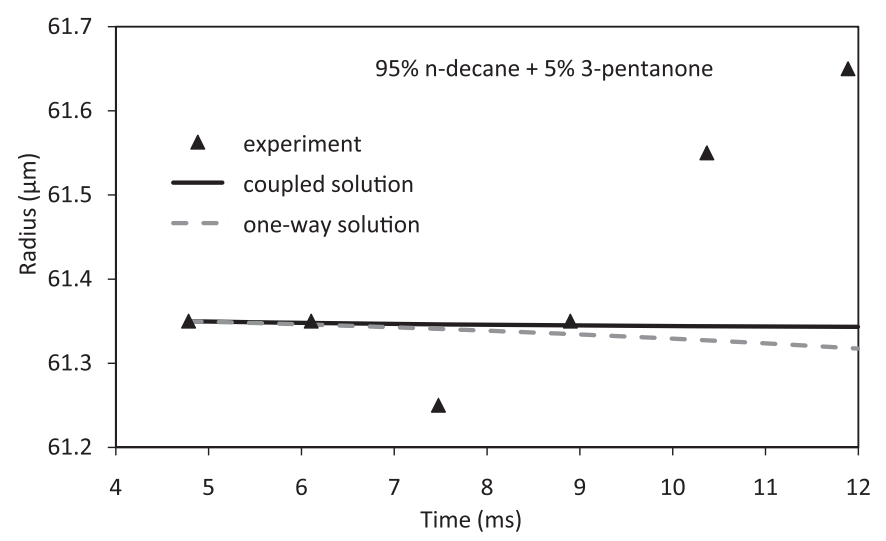

Fig. 6. The plots of $R_{d}$ versus time predicted by the one-way and coupled solutions for the same conditions as in Fig. 2.

solutions, is presented in Fig. 9. As can be seen from this figure, at all times the temperature increases with the distance from the centre of the droplets, as expected. The temperature gradient is less important for case predicted by the coupled solution compared with the one-way solution. The deviation between the temperatures in all areas of the droplets, predicted by coupled and one-way solutions, increases with time.

The plots similar to those shown in Fig. 9, but for the mass fraction of $n$-decane, are presented in Fig. 10. As can be seen from this figure, the mass fraction of n-decane in the areas close to the surface of the droplet, predicted by coupled and on-way solutions, increases with time. This is related to higher volatility of 3-pentanone, compared with n-decane. As in the case of temperature, shown in Fig. 9, the rate of increase of the mass fraction of n-decane, predicted by the coupled solution, is slower than the one predicted by the one-way solution. The deviation between the results predicted by these solutions increases with time. This can be related to the fact that in the coupled solution, vapour in the region of influence is partially saturated and the temperature in this region is lower than that in the ambient gas.

The time evolution of evaporated masses of 3-pentanone, $\mathrm{n}$-decane and the total vapour mass in the region of influence is shown in Fig. 11. As one can see from this figure, evaporated masses of both substances increase with time. This can be related to the fact that no condensation takes place during the period under consideration, and to our assumption that no vapour escapes from the region of

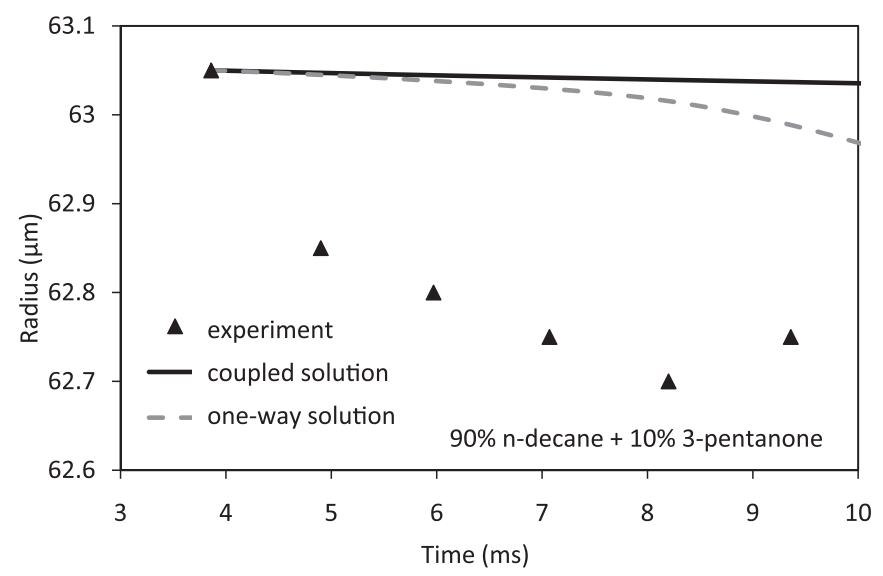

Fig. 7. The plots of $R_{d}$ versus time predicted by the one-way and coupled solutions for the same conditions as in Fig. 3.

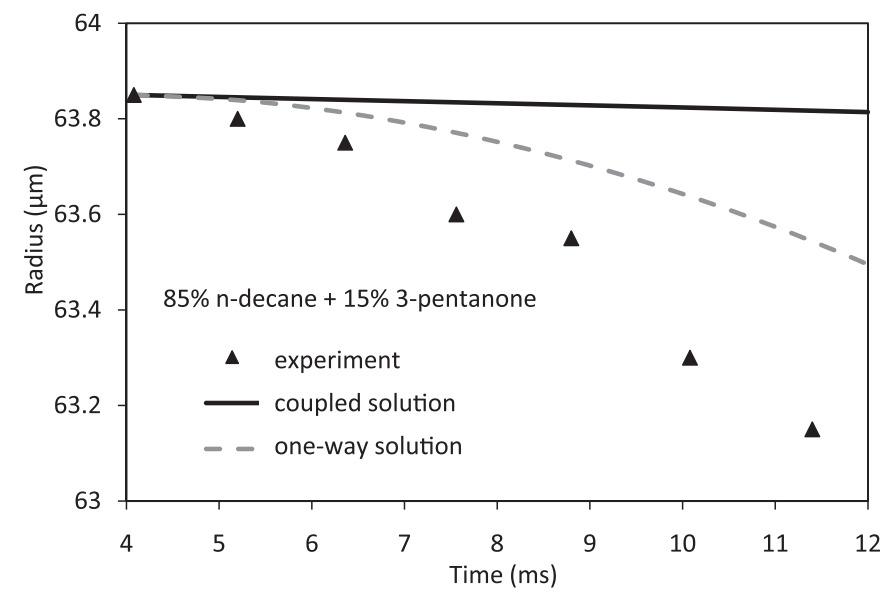

Fig. 8. The plots of $R_{d}$ versus time predicted by the one-way and coupled solutions for the same conditions as in Fig. 4.

influence into ambient gas. The predicted mass of n-decane is always greater than that of 3-pentanone, despite higher volatility of 3-pentanone, compared with n-decane (cf. Fig. 10). This is related to the fact that the original amount of n-decane in droplets is 20 times more than the amount of 3-pentanone.

The time evolution of masses of air and the total mass of the mixture of air and vapour in the region of influence is shown in Fig. 12. The increase of the mass of air with time is related to the decrease of temperature in the interaction volume with time (cf. Fig. 5) at constant pressure. Note that for a fixed temperature, part of air should have been removed from the interaction volume by the evaporating fuel (see Eq. (31)).

\section{Accuracy versus CPU efficiency}

In the analysis, reported in our earlier paper [9], we were concerned with the accuracy of the results, and chose the maximum number of terms in the series in the analytical solutions for temperature and species mass fractions. However, remembering that perhaps the most important practical application of the model described in [9] would be its potential implementation into computational fluid dynamic (CFD) codes, and application of the latter to simulation of realistic engineering processes, finding a reasonable compromise between the accuracy of the model and

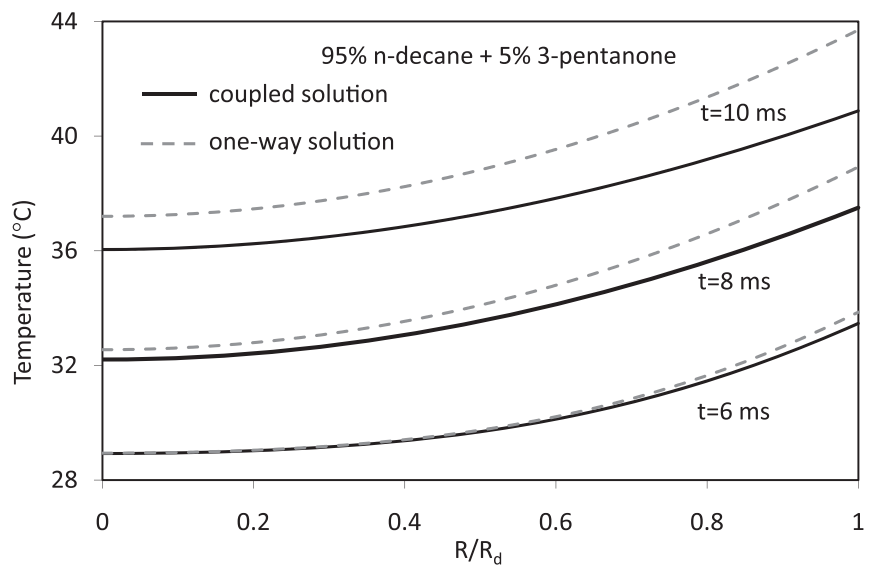

Fig. 9. The plots of $T$ versus $R / R_{d}$ for three moments of time after the start of calculations predicted by the one-way and coupled solutions and for the same conditions as in Fig. 2. 


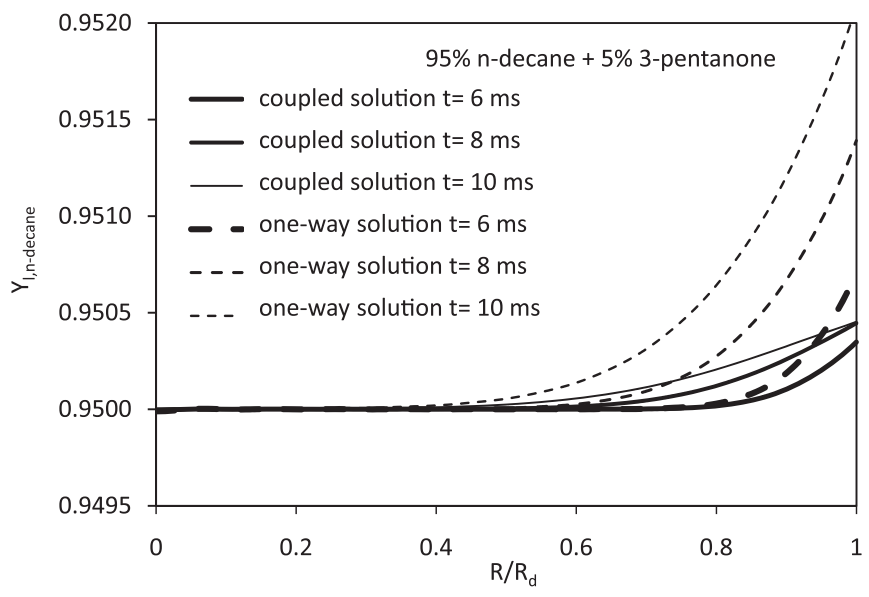

Fig. 10. The plots of the $n$-decane mass fraction $Y_{l \mathrm{n} \text {-decane }}$ versus $R / R_{d}$ for three moments of time after the start of calculations predicted by the one-way and coupled solutions and for the same conditions as in Fig. 2.

its CPU efficiency becomes more important than its accuracy alone. The accuracy and CPU efficiency of the model, depending on the number of terms in the series in the analytical solutions for temperature and species mass fractions, is investigated in this section. We focus on the analysis of the 95\% decane - 5\% 3pentanone mixture droplets (Case 1 ). The conclusions referring to other mixtures are essentially the same as the ones for the abovementioned mixture.

In our previous paper [34] we have shown that modelling of mono-component droplet heating and evaporation, based on the analytical solution of the heat conduction equation inside the droplet, is more CPU efficient and accurate than when based on the numerical solution of this equation. We anticipate that the same conclusion remains valid for multi-component droplets of the type considered in our paper, when the species diffusion inside droplets is taken into account.

The relative errors were estimated based on the following equation:

Error $=\frac{\sum_{i=1}^{i=i_{\max }}\left(\left|F_{N=n}-F_{N=100}\right| / F_{N=100}\right)}{i_{\max }}$,

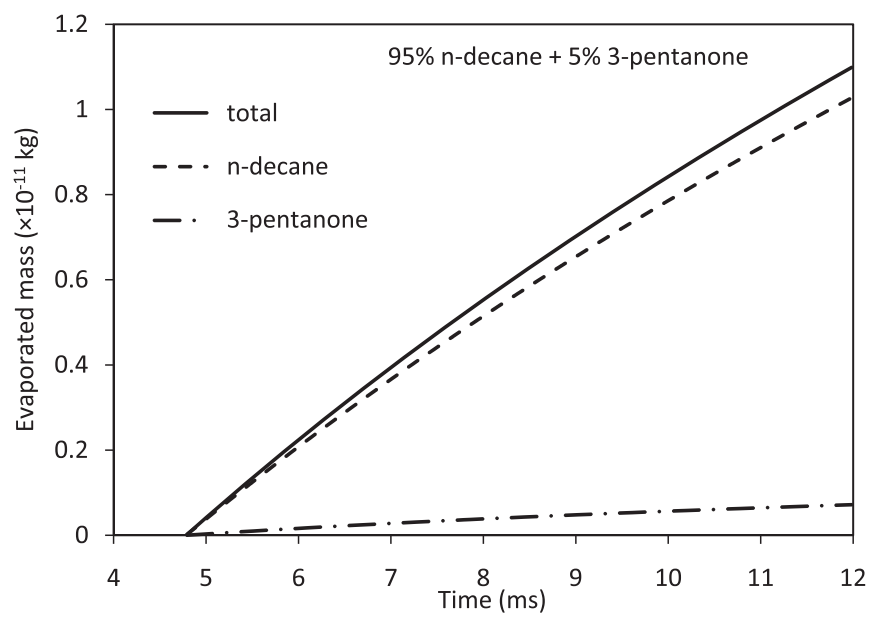

Fig. 11. The plots of the evaporated masses of n-decane, 3-pentanone and the total evaporated mass versus time predicted by the coupled solution for the same conditions as in Fig. 2

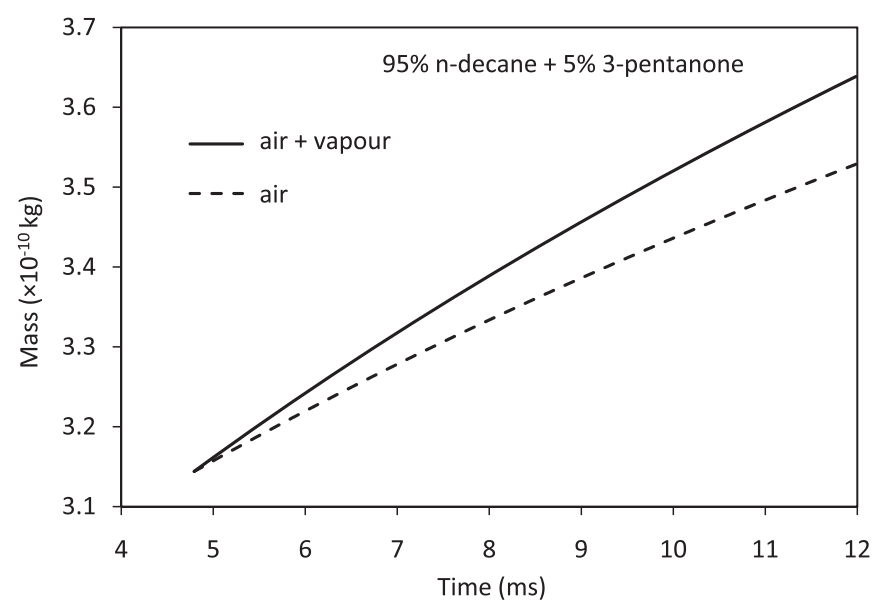

Fig. 12. The plots of the total mass of air and the mixture of air and vapour in the region of influence versus time predicted by the coupled solution for the same conditions as in Fig. 2.

where $F$ stands for either temperature or mass fraction, $N$ is the number of terms in the series in the solution to the equation for temperature or mass fraction, $i$ refers to time steps, $i_{\max }$ is the maximal number of timesteps used in calculation (this number varied from about 700 to about 800 ). The errors were calculated relative to the values obtained when 100 terms in the expression for the temperature or mass fraction of n-decane were used. Higher order terms did not produce any effects on the results.

The plots of relative errors of the temperatures at the centre of the droplets $\left(T_{C}\right)$, the surface of the droplets $\left(T_{S}\right)$, the average droplet temperatures $\left(T_{a v}\right)$, predicted by the coupled solution, and the CPU requirement versus the number of terms in the series in the expression for droplet temperature $(n)$ are presented in Fig. 13. 100 terms in the expression for the mass fraction of n-decane (Eq. (79) in Appendix 2) were taken. All temperatures were measured in ${ }^{\circ} \mathrm{C}$. Calculations were performed on $3000 \mathrm{~Hz}$ CPU, 3 GB RAM work station. As follows from this figure, the errors of calculating all temperatures, are practically equal to zero when the number of terms $n$ is close or larger than 20. Hence, 20 terms were used in the analysis presented in Section 6 . The CPU time increased by about $50 \%$ when the number of terms in the expression for temperature increased from 20 to 100 . This increase of the number of terms, however, does not lead to any increase in the accuracy of the results

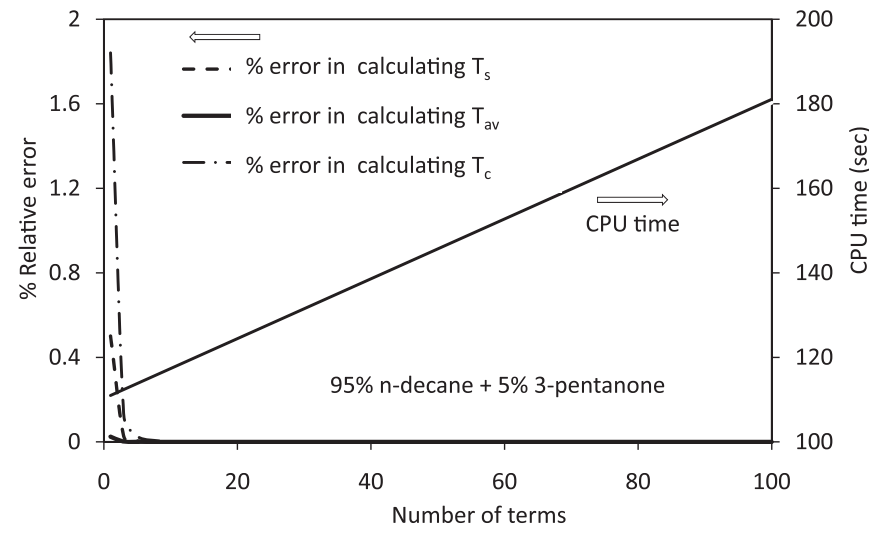

Fig. 13. The plots of relative errors (in percent) of calculation of droplet surface, average and central temperatures $\left(T_{s}, T_{a v}\right.$ and $\left.T_{c}\right)$ and $\mathrm{CPU}$ time versus the number of terms in the solution to the temperature equation with the fixed number of terms in the solution to the species equation equal to 100 for the same conditions as in Fig. 2. 
and cannot be justified. Note that the errors of calculating average temperature are more than an order of magnitude less than the errors of calculating droplet surface temperature and the temperatures at the centre of the droplets. This is related to the fact that for small number of terms, small oscillations of temperature were observed near the centre of the droplet and its surface. These oscillations reduce substantially during the integration along $R$ performed for the calculation of the droplet average temperature.

The plots similar to those presented in Fig. 13, but for the mass fractions of n-decane, are shown in Fig. 14. In contrast to Fig. 13, the number of terms in the series in Eq. (79) varied, and the number of terms in the expression for the temperature was assumed to be equal to 100 . As can be seen from this figure, all errors of calculation of the mass fractions of $n$-decane are about two orders of magnitude less than the errors of calculation of droplet temperatures. As in the case of temperatures, the errors of calculating average mass fractions are more than an order of magnitude less than the errors of calculating the mass fractions at the surface and the centre of the droplets. The explanation of this phenomenon is the same as for the droplet temperature (see Fig. 13). As in the case of temperature, the errors in the case when the number of terms are about or more than 20 , can be safely ignored. The increase of CPU time when the number of terms increased from 20 to 100 , was even more noticeable than in the case of temperature (this time almost doubled). As in the case of temperature, this increase in the number of terms could not be justified from the point of view of increased accuracy of the model.

The plots similar to those shown in Fig. 13 but assuming that the numbers of terms in the series for $T$ and mass fractions are equal are presented in Fig. 15. We focus on the case when these numbers are less than 20. As can be seen from this figure, the errors of estimating all temperatures are less than about $0.5 \%$, even if the number of terms in both series is reduced to just three. At the same time the CPU time, in the case when three terms are chosen, is more than an order of magnitude less that in the case when hundred terms is chosen in both series.

The plots similar to those shown in Fig. 14, but referring to the case when the numbers of terms in the series for $T$ and mass fractions are equal, are presented in Fig. 16. As can be seen from this figure, the errors of estimating all mass fractions are less than about $0.01 \%$ even if the number of terms in both series is reduced to just three. Note that the increase in the number of these terms from five to twenty practically does not improve the accuracy of the prediction of the model. As in the case of the results shown in Fig. 15, the CPU time, in the case when three terms are chosen, is

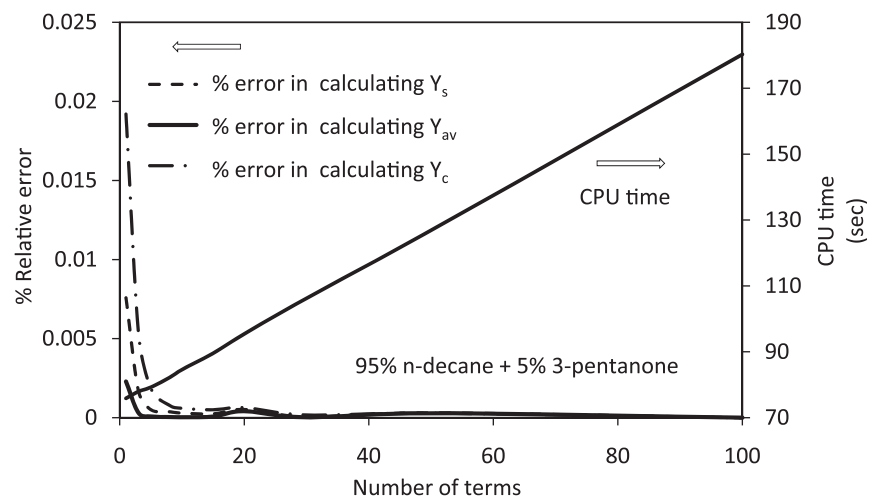

Fig. 14. The plots of relative errors (in percent) of calculation of liquid n-decane mass fractions at the surface of the droplet, average mass fraction of n-decane and its mass fraction at the centre of the droplet $\left(Y_{s}, Y_{a v}\right.$ and $\left.Y_{c}\right)$ and CPU time versus the number of terms in the solution to the species equation with fixed number of terms in the solution to the temperature equation equal to 100 for the same conditions as in Fig. 2 .

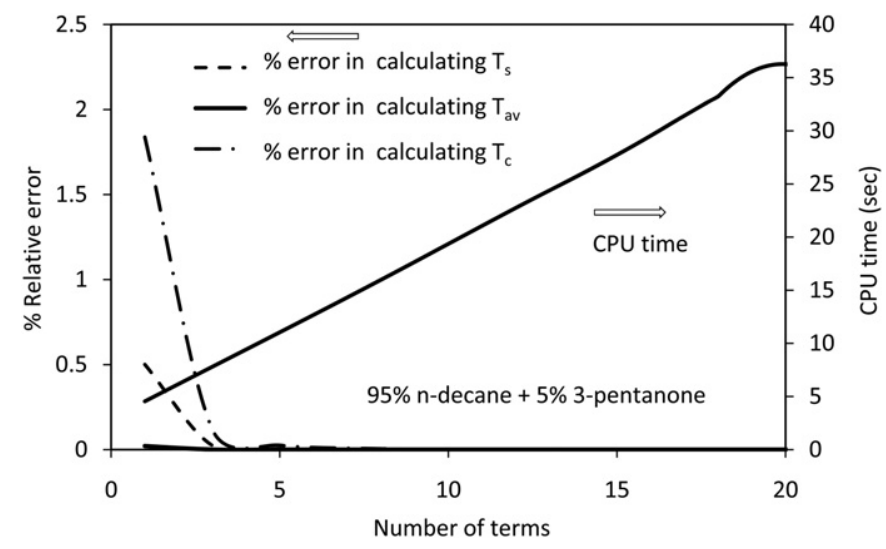

Fig. 15. The plots of errors (in percent) of calculation of droplet surface, average and central temperatures $\left(T_{s}, T_{a v}\right.$ and $\left.T_{c}\right)$ and CPU time versus the number of terms in the solutions to the temperature and species equations (these numbers are assumed equal) for the same conditions as in Fig. 2.

more than an order of magnitude less than in the case when a hundred terms is chosen in both series.

Combining the results shown in Figs. 13-16, the model can accurately predict the values of droplet temperature and mass fractions of $n$-decane even if the number of terms in the series for temperature and mass fractions is reduced to three. This result is consistent with the earlier finding reported in [22]. Relatively low CPU time required for calculations makes this model potentially attractive for implementation in CFD codes.

As mentioned earlier, all plots presented so far refer to the case when the time step was chosen equal to $10^{-5} \mathrm{~s}$ and the number of points along the radius, $N_{R}$, equal to 2000 . The increase in the time step is expected to decrease the accuracy of calculations as in any numerical code. In our case, however, the excessive reduction of this time step can also lead to decrease in the accuracy of calculations due to the decrease in the Fourier numbers in the series in the analytical expressions for $T$ and $Y_{l i}$. This decrease is expected to affect the convergence of these series. This effect is illustrated in Fig. 17, where the plots of droplet surface temperatures versus $N_{R}$ for different timesteps are presented for the $95 \%$ n-decane - 5\% 3pentanone mixture (Case 1) $5 \mathrm{~ms}$ after the start of calculations. As follows from this figure, the choice of the time step $10^{-3} \mathrm{~s}$ leads to under-prediction of this temperature by more than $1{ }^{\circ} \mathrm{C}$ for all $N_{R}$.

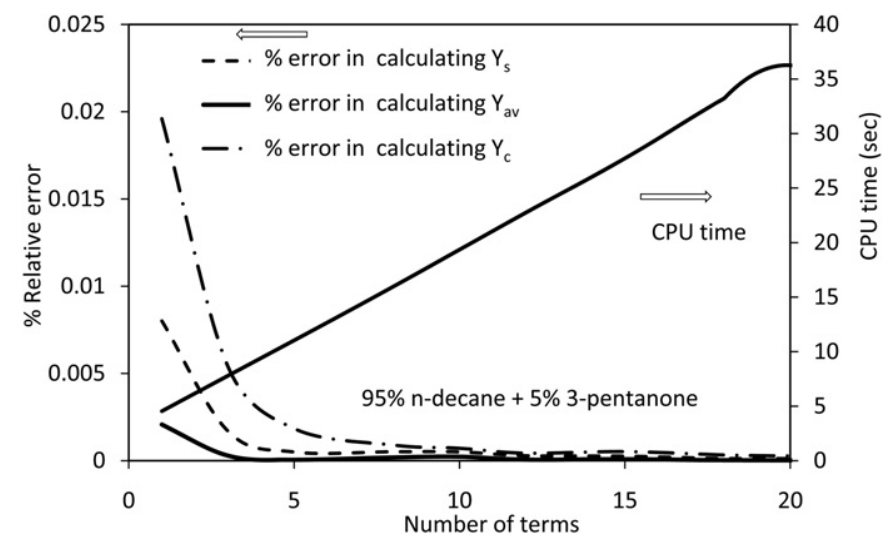

Fig. 16. The plots of errors (in percent) of calculation of liquid n-decane mass fractions at the surface of the droplet, average mass fraction and the mass fraction at the centre of the droplet $\left(Y_{S}, Y_{a v}\right.$ and $\left.Y_{c}\right)$ and CPU time versus the number of terms in the solutions to the temperature and species equations (these numbers are assumed equal) for the same conditions as in Fig. 2 . 


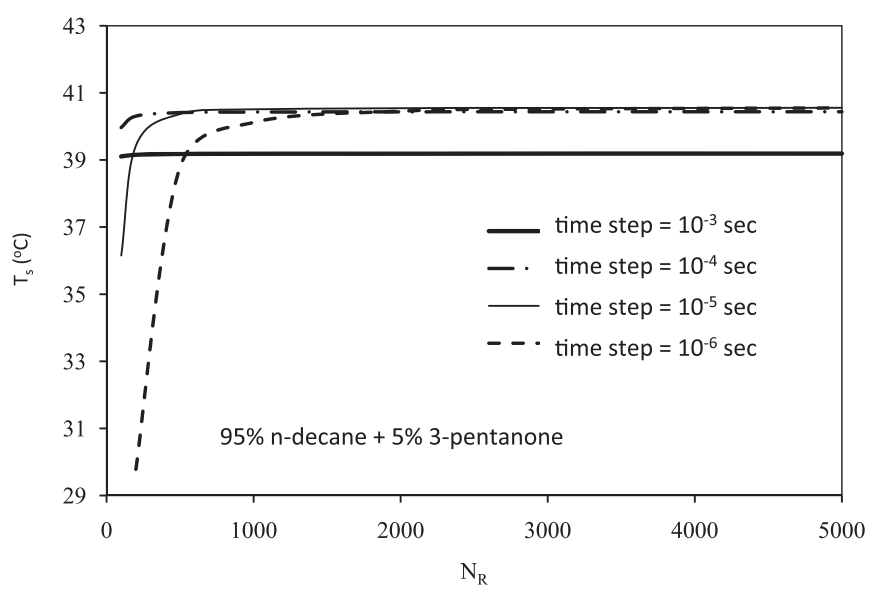

Fig. 17. The plots of the predicted droplet surface temperatures versus $N_{R}$ for different timesteps for the $95 \%$ n-decane - 5\% 3-pentanone mixture (Case 1) at $5 \mathrm{~ms}$ after the start of calculations.

For all timesteps in the range $10^{-6}-10^{-4} \mathrm{~s}$ and $\mathrm{NR} \geq 1500$ the predicted temperature remains almost the same. For smaller $N_{R}$, the accuracy of calculations clearly deteriorates, especially for the time step equal to $10^{-6} \mathrm{~s}$. The plots, shown in Fig. 17, are similar to those obtained for other cases and other moments of time (these are not shown in the paper). Based on these plots, the values of the time step $10^{-5} \mathrm{~S}$ and $N_{R}=2000$ have been chosen. Note that the CPU time could be further reduced, practically without any detrimental effects on accuracy, if the time step is increased to $10^{-4} \mathrm{~s}$ and $N_{R}$ is reduced to 1000 .

Note that although the numerical code, used in our analysis, is based on the analytical solutions both for temperature and species mass fractions, it still requires the introduction of time steps and the spatial mesh inside the droplets. The time steps are required to take into account the time dependence of gas temperature in the zone of influence and the evaporation process. The spatial mesh is required for calculating the integrals used in the analytical solutions, the averaging of variables and postprocessing.

\section{Conclusions}

The earlier reported simplified model for multi-component droplet heating and evaporation is generalised to take into account the coupling between the droplets and the ambient gas. Similarly to the original simplified model, the model takes into account droplet heating by convection from the ambient gas, the distribution of temperature inside the droplet, diffusion of liquid species inside the droplet, droplet swelling or contraction due to changing average temperature, the effects of recirculation in the moving droplets on heat and species mass diffusion within them. The effects of the nonunity activity coefficient are ignored (the Raoult law is assumed to be valid) and the interaction between droplets is taken into account based on the correlation suggested in [20]. The size of the gas volume, where the interaction between droplets and gas needs to be taken into account (region of influence), is estimated based on the characteristic thermal and mass diffusion scales.

The model is applied to the analysis of the experimentally observed heating and evaporation of monodispersed n-decane/3pentanone mixture droplets at atmospheric pressure. It is pointed out that the effect of coupling leads to noticeably better agreement between the predictions of the model and the experimentally observed average droplet temperatures. In most cases, the experimentally observed droplet temperatures lie between the average and central temperatures, predicted by the coupled solution. The main effect of the coupled solution is linked with the reduction of the gas temperature in the region of influence. A deviation of up to about $0.5 \mu \mathrm{m}$ between the experimentally observed and predicted droplet radii (less than $1 \%$ ) is related to the experimental margins. It is pointed out that the observed time evolution of droplet radii cannot be used for the validation of the model.

It is pointed out that the number of terms in the series in the expressions for droplet temperature and species mass fraction can be reduced to just three, with possible errors less than about $0.5 \%$. In this case the model can be recommended for the implementation into computational fluid dynamics (CFD) codes and used for various engineering applications, including those in internal combustion engines.

\section{Acknowledgements}

The authors are grateful to the European Regional Development Fund Franco-British INTERREG IVA (Project C5, Reference 4005) for the financial support of this project.

\section{Appendix 1}

\section{Transport coefficients for mixtures}

\section{Liquid thermal conductivity for a mixture}

A number of approximate formulae for thermal conductivity in multi-component liquids are reviewed in [35]. We use the Vredeveld equation, valid for nonaqueous systems in which the ratio of thermal conductivities does not exceed two [35]:

$k_{l}=\left(\sum_{i} Y_{i} k_{l i}^{-2}\right)^{-1 / 2}$,

where $Y_{i}$ are mass fractions for species $i, k_{l i}$ are thermal conductivities of species $i$.

\section{Liquid viscosity for a mixture}

A number of approximate formulae for dynamic viscosity of multi-component liquids $\left(\mu_{l}\right)$ are reviewed in [35]. It was shown that in the case of two species, the approach suggested by Grunberg and Nissan [36] is the most accurate and convenient in practical applications:

$\ln \mu_{l}=\sum_{i=1}^{2} X_{i} \ln \mu_{l i}+X_{1} X_{2} G_{12}$,

where $G_{12}$ can be negative or positive.

As follows from the example presented in [36], the contribution of the term proportional to $G_{12}$ in this equation is about $1 \%$. In our analysis the liquid viscosity is used for the estimate of the correction factors in effective thermal conductivity and effective diffusivity models. These correction factors are rather weak functions of liquid viscosity especially for large Peclet numbers. This allows us to ignore the term proportional to $G_{12}$, and reduce the above equation to the Arrhenius formula [36]

$\ln \mu_{l}=\sum_{i=1}^{2} X_{i} \ln \mu_{l i}$

In the case of several components, this equation can be generalised to

$\ln \mu_{l}=\sum_{i} X_{i} \ln \mu_{l i}$

The latter equation is used in our analysis. 
Liquid diffusivity for a mixture

In the case of two-component droplets it was suggested that the liquid diffusivity of the mixture is estimated based on the following simplified formula [9]:

$D_{12}=X_{1} D_{12}^{0}+X_{2} D_{21}^{0}$

where $D_{12}^{0}$ and $D_{21}^{0}$ are diffusivities of dilute solute 1 in solvent 2 , and dilute solute 2 in solvent 1 respectively.

In the case when three or more components are present in the droplets, the situation becomes much more complex as the diffusion flux of each species depends not only on the concentration gradient of the species in question, but also on concentration gradients of other species. In this case, a single diffusion coefficient, describing the diffusion of a given type of species, as defined by Eq. (36), needs to be replaced by $N-1$ diffusion coefficients, where $N$ is the number of species. These are the self diffusion coefficient and cross-diffusion coefficients [35]. The analysis of this diffusion process can be simplified in the case when the concentration of a given species is low (solute) [35], which is generally not the case when realistic multi-component droplets are considered.

We suggest the following approach allowing us to describe the diffusion of individual species $i$ in $\mathrm{N}$-component droplets based on a single diffusion coefficient $D_{i m}$. In this approach we consider the mixture of all species, except $i$ as the second component in the droplet, and the problem of diffusion in a multi-component droplet is reduced to the problem of diffusion in a two-component droplet which was already considered in [9], when $X_{m}=\sum_{j \neq i} X_{j}$. Without loss of generality we assume that $i=1$ and $X_{m}=\sum_{j=2}^{N} X_{j}$.

Now we assume that the sum of all components, except the first one, is replaced by the component 2 , with the molar fraction equal to $X_{m}$. In this case we can calculate $D_{12}$ as:

$D_{12}=X_{1} D_{12}^{0}+X_{m} D_{21}^{0}$,

Then we assume that the sum of all components, except the first one, is replaced by the component 3 , with the molar fraction equal to $X_{m}$. In this case we can calculate $D_{13}$ as:

$D_{13}=X_{1} D_{12}^{0}+X_{m} D_{31}^{0}$,

Following the same procedure, we assume that the sum of all components, except the first one, is replaced by the components 4 , $5, \ldots, N$, with the molar fraction equal to $X_{m}$. In this case we can calculate $D_{1 N}$ as:

$D_{1 N}=X_{1} D_{12}^{0}+X_{m} D_{N 1}^{0}$.

The value of $D_{1 m}$ is calculated as

$D_{1 m}=\left(\sum_{j=2}^{N} X_{j} D_{1 j}\right) /\left(\sum_{j=2}^{N} X_{j}\right)$,

where $D_{1 j}$ are obtained based on Eqs. (37)-(39). The diffusion coefficients for all other components are calculated in the same way.

We cannot justify rigorously this approach at the moment, except that it gives a correct result in the limit when one component in the mixture $j=2,3 \ldots, N$ dominates over others. It is expected to predict the correct order of magnitude of this coefficient if this is not the case. This approach is more general than the one used in [37], where it was assumed that the diffusion coefficients of all species are the same (see Eq. (3.5a) in this book).
Gas diffusivity for a mixture

In the case of mono-component droplets, the value of the diffusion coefficient of vapour in air $D_{v a}$ can be estimated from the Wilke and Lee formula [35]:

$D_{v a}=\frac{\left[3.03-\left(0.98 / M_{v a}^{1 / 2}\right)\right]\left(10^{-7}\right) T^{3 / 2}}{p M_{v a}^{1 / 2} \sigma_{v a}^{2} \Omega_{D}}$,

where $D_{v a}$ is in $\mathrm{m}^{2} / \mathrm{s}, T$ is temperature in $\mathrm{K}$,

$M_{v a}=2\left[\left(1 / M_{v}\right)+\left(1 / M_{\mathrm{air}}\right)\right]^{-1}$

$M_{v}$ and $M_{\mathrm{air}}$ are molar masses of vapour and air respectively, $p$ is pressure in bar, $\sigma_{\mathrm{va}}=\left(\sigma_{v}+\sigma_{a}\right) / 2, \sigma_{v}$ and $\sigma_{a}$ are characteristic Lennard-Jones lengths for vapour and air respectively, measured in Angstrom (A), $\Omega_{D}$ is the function of the normalised temperature $T^{*}=$ $k_{B} T / \varepsilon_{v a}$ given by Eq. (B6) in [38], $\varepsilon_{v a}=\sqrt{\varepsilon_{v} \varepsilon_{a}}, \varepsilon_{v}$ and $\epsilon_{a}$ are characteristic Lennard-Jones energies for vapour and air respectively, $k_{B}$ is the Boltzmann constant

In the case of the mixture of vapour components, the crossdiffusion coefficients $D_{i j}$ would have to be estimated [35]. The analysis of this process can be substantially simplified if we assume that the diffusing component $i$ is a dilute one and the remaining mixture is homogeneous. In this case the value of the diffusion coefficient of this component in the mixture $\left(D_{i m}\right)$ can be obtained from the Blanc law [35]:

$D_{i m}=\left(\sum_{j=1 ; j \neq i}^{N} \frac{X_{j}}{D_{i j}}\right)^{-1}$

If air dominates over all vapour components then this equation reduces to the statement that $D_{i m}=D_{i a}$.

However, even in this simplified case, the problem of the diffusion of multi-component vapour in air remains rather complex. To simplify the problem further, the multi-component vapour will be treated as mono-component (similarly to the case of air). In this case the value of $D_{v a}$ will be calculated based on Eq. (41) in which all input parameters (molar masses and Lennard-Jones parameters) are calculated as molar averaged, taking into account their relative molar concentrations.

This approach can be justified by the fact that for heavy hydrocarbons the value of $M_{v a}$ is mainly controlled by air, while the difference in the values of $\sigma_{v}$ for various components in most cases differ not more than by about $20 \%$.

\section{Gas thermal conductivity and viscosity}

The thermal conductivity $\left(k_{v}\right)$ and dynamic viscosity $\left(\mu_{v}\right)$ for the mixture of air and fuel vapour was calculated following the procedure described in [33].

\section{Appendix 2}

Solution of Eq. (15)

In what follows, the solution of Eq. (15) $\left(Y_{l i}(t, R)\right)$ for $t \geq 0$ and 0 $\leq R<R_{d}$ is found. Remembering the physical background of the problem, we will look for a solution which is continuously differentiable twice in the whole domain.

Let us rewrite the boundary condition (16) in the form:

$\left.\left(\frac{\partial Y_{l i}}{\partial R}-\frac{\alpha}{D_{l}} Y_{l i}\right)\right|_{R=R_{d}}=-\frac{\alpha \epsilon_{i}(t)}{D_{l}}$.

The initial condition is $Y_{l i}(t=0)=Y_{l i 0}(R)$.

We look for the solution to Eq. (15) in the form: 
$Y_{l i}(t, R)=y(t, R)+\epsilon(t)$,

where the subscript $i$ at $\epsilon$ is omitted to simplify the notation.

Having substituted (43) into Eq. (15) and the boundary condition (42) we can rewrite this equation and the corresponding boundary and initial conditions in the form:

$\frac{\partial y}{\partial t}=D_{l}\left(\frac{\partial^{2} y}{\partial R^{2}}+\frac{2}{R} \frac{\partial y}{\partial R}\right)-\frac{\mathrm{d} \epsilon(t)}{\mathrm{d} t}$,

$\left.\left(\frac{\partial y}{\partial R}-\frac{\alpha}{D_{l}} y\right)\right|_{R=R_{d}}=0$

$\left.y\right|_{t=0}=Y_{l i 0}(R)-\epsilon(0) \equiv Y_{l i 0}(R)-\epsilon_{0}$.

Introduction of the new variable, following [39],

$u(t, R)=y(t, R) R$

allows us to rewrite Eq. (44) and the corresponding boundary and initial conditions in the form:

$\frac{\partial u}{\partial t}=D_{l} \frac{\partial^{2} u}{\partial R^{2}}-R \frac{\mathrm{d} \epsilon(t)}{\mathrm{d} t}$,

$\left.u\right|_{R=0}=\left.\left(\frac{\partial u}{\partial R}+\frac{h_{0}}{R_{d}} u\right)\right|_{R=R_{d}}=0$,

$\left.u\right|_{t=0}=R\left(Y_{l i 0}(R)-\epsilon_{0}\right)$,

where:

$h_{0}=-\left(1+\frac{\alpha R_{d}}{D_{l}}\right)$.

Note that the change of the variable from $y$ to $u$ leads to the need for a second boundary condition at $R=0$. Our assumption that the solution is continuously differentiable twice implies that $y$ is finite everywhere in the domain $0 \leq R<R_{d}$. Hence, the boundary condition (48) at $R=0$. The solution to the problem (47)-(49) for $h_{0}>-1$ was earlier reported in [39]. Here the focus will be on the case $h_{0}<-1$, which is directly relevant to the problem of diffusion of species inside droplets.

We look for the solution to Eq. (47) in the form:

$u=\sum_{n=0}^{\infty} \Theta_{n}(t) v_{n}(R)$

where $v_{n}(R)$ is the full set of non-trivial solutions to the equation:

$\frac{\partial^{2} v}{\partial R^{2}}+p v=0$

subject to the boundary conditions:

$\left.v\right|_{R=0}=\left.\left(\frac{\partial v}{\partial R}+\frac{h_{0}}{R_{d}} v\right)\right|_{R=R_{d}}=0$.

Eq. (51) with boundary conditions (52) is the well known Sturm-Liouville problem. Our first task is to find eigenvalues $p$ for this problem. The cases $p=0, p<0$ and $p>0$ will be considered separately.

The Sturm-Liouville problem for $p=0$

For $p=0$ the general solution to Eq. (51) can be presented as $v=A+B R$. The condition $\left.v\right|_{R=0}=0$ implies that $A=0$. The boundary condition at $R=R_{d}$ leads to the following equation
$B\left(1+h_{0}\right)=0$. Since $h_{0} \in(-\infty,-1)$, the latter equation is satisfied only when $B=0$. This leads to the trivial solution $v=0$ which is disregarded in our analysis. Hence, Eq. (51) has no non-trivial solutions for $p=0$.

The Sturm-Liouville problem for $p<0$

Assuming that $p=-\lambda^{2}<0$ we write the general solution to (51) as:

$v(R)=A \cosh \left(\lambda \frac{R}{R_{d}}\right)+B \sinh \left(\lambda \frac{R}{R_{d}}\right)$,

where $A$ and $B$ are arbitrary constants.

The boundary condition at $R=0$ (see (52)) implies that $A=0$. The boundary condition at $R=R_{d}$ leads to the following equation:

$\frac{B}{R_{d}}\left(\lambda \cosh \lambda+h_{0} \sinh \lambda\right)=0$.

$B$ in this equation is not equal to zero as we do not consider the trivial solution $v=0$. Hence, Eq. (54) can be re-written as:

$\tanh \lambda=-\frac{\lambda}{h_{0}}$

It is easy to show that for $h_{0} \in(-\infty,-1)$ Eq. (55) has three solutions $\lambda=0 ; \pm \lambda_{0}$, where $\lambda_{0} \in(0,+\infty)$, and it has no solutions for $h_{0}>-1$. The solution $\lambda=0$ leads to the trivial solution $v=0$, which is disregarded in our analysis. The solutions $\lambda= \pm \lambda_{0}$ lead to solutions (53) (eigen functions) which differ only by the sign of $B$. Since the value of the coefficient $B$ is determined by the normalisation condition only (see below), the solution $\lambda=-\lambda_{0}$ can be disregarded. Hence, we can conclude that the solution of Eq. (55) gives only one eigenvalue $\lambda=\lambda_{0}>0$ and the corresponding eigen function

$v_{0}(R)=\sinh \left(\lambda_{0} \frac{R}{R_{d}}\right)$,

where the normalisation leading to $B=1$ has been chosen.

The direct calculation of the integrals, taking into account condition (55), leads to the following expression for the norm of $v_{0}$ :

$\left\|v_{0}\right\|^{2}=\int_{0}^{R_{d}} v_{0}^{2}(R) \mathrm{d} R=-\frac{R_{d}}{2}\left[1+\frac{h_{0}}{h_{0}^{2}-\lambda_{0}^{2}}\right]$.

The Sturm-Liouville problem for $p>0$

Assuming that $p=\lambda^{2}>0$ we write the general solution to (51) as:

$v(R)=A \cos \left(\lambda \frac{R}{R_{d}}\right)+B \sin \left(\lambda \frac{R}{R_{d}}\right)$,

where $A$ and $B$ are arbitrary constants.

The boundary condition at $R=0$ (see (52)) implies that $A=0$. The boundary condition at $R=R_{d}$ leads to the following equation:

$\frac{B}{R_{d}}\left(\lambda \cos \lambda+h_{0} \sin \lambda\right)=0$.

$B$ in this equation is not equal to zero as we do not consider the trivial solution $v=0$. Hence, Eq. (59) can be re-written as:

$\tan \lambda=-\frac{\lambda}{h_{0}}$

As in the case $p<0$ we disregard the solutions to this equation corresponding to zero and negative $\lambda$. A countable set of positive 
solutions to this equation (positive eigenvalues) $\lambda_{n}$ are arranged in ascending order:

$0<\lambda_{1}<\lambda_{2}<\lambda_{3}<\ldots$

The corresponding eigen function can be presented as:

$v_{n}(R)=\sin \left(\lambda_{n} \frac{R}{R_{d}}\right)$,

where the normalisation leading to $B=1$ has been chosen as in the case $p<0$.

The direct calculation of the integrals, taking into account Condition (60), leads to the following expression for the norm of $v_{n}$ for $n \geq 1$ :

$\left\|v_{n}\right\|^{2}=\int_{0}^{R_{d}} v_{n}^{2}(R) \mathrm{d} R=\frac{R_{d}}{2}\left[1+\frac{h_{0}}{h_{0}^{2}+\lambda_{0}^{2}}\right]$.

The norm (62) differs from the norm chosen in [39] by the factor $R_{d}$ (see their Eq. (A4)). This does not affect the final solution.

Orthogonality of the eigen functions

The orthogonality of functions $v_{n}(n \geq 1)$ was shown in our previous paper [39]. To show that functions $v_{0}$ and $v_{n}(n \geq 1)$ are orthogonal, we need to calculate the following integral:

$I=\int_{0}^{R_{d}} \sinh \left(\lambda_{0} \frac{R}{R_{d}}\right) \sin \left(\lambda_{n} \frac{R}{R_{d}}\right) \mathrm{d} R$,

where $n \geq 1$.

Using integration by parts twice when calculating the integral on the right hand side of Eq. (63) we rearrange this equation to:

$I=-\frac{R_{d}}{\lambda_{n}}\left[\sinh \lambda_{0} \cos \lambda_{n}-\frac{\lambda_{0}}{\lambda_{n}} \cosh \lambda_{0} \sin \lambda_{n}+\frac{\lambda_{0}^{2}}{\lambda_{n} R_{d}} I\right]$

where $I$ in the right hand side of this equation is the same as in (63).

Eq. (64) can be rearranged to:

$I=-\frac{\frac{R_{d}}{\lambda_{n}}\left[\frac{\sinh \lambda_{0}}{\cosh \lambda_{0}}-\frac{\lambda_{0}}{\lambda_{n}} \frac{\sin \lambda_{n}}{\cos \lambda_{n}}\right] \cosh \lambda_{0} \cos \lambda_{n}}{1+\left(\frac{\lambda_{0}}{\lambda_{n}}\right)^{2}}$.

Remembering Eqs. (55) and (60), we can see that $I$ defined by Eq. (65) is equal to zero. This implies that functions $v_{n}$ are orthogonal for $n \geq 0$ and we can write:

$\int_{0}^{R_{d}} v_{n}(R) v_{m}(R) \mathrm{d} R=\delta_{n m}\left\|v_{n}\right\|^{2}$,

where $n \geq 0$ and $m \geq 0,\left\|v_{n}\right\|^{2}$ is defined by (57) when $n=0$ and (62) when $n \geq 1$.

Expansion of $R$ in a Fourier series with respect to functions $v_{n}$

Before we are able to use (50) for the solution of Eq. (47) we need to find the presentation of $R$ as a Fourier series with respect to functions $v_{n}$ :

$R=\sum_{n=0}^{\infty} Q_{n} v_{n}(R)$, where

$Q_{n}=\frac{1}{\left\|v_{n}\right\|^{2}} \int_{0}^{R_{d}} R v_{n}(R) \mathrm{d} R$.

Direct calculation of the integrals in the right hand side of (68) leads to the following explicit expressions for $Q_{n}$ :

$Q_{n}=\left\{\begin{array}{ccc}-\frac{1}{\left\|v_{0}\right\|^{2}}\left(\frac{R_{d}}{\lambda_{0}}\right)^{2}\left(1+h_{0}\right) \sinh \lambda_{0} & \text { when } & n=0 \\ \frac{1}{\left\|v_{n}\right\|^{2}}\left(\frac{R_{d}}{\lambda_{n}}\right)^{2}\left(1+h_{0}\right) \sin \lambda_{n} & \text { when } & n \geq 1\end{array}\right.$

Calculation of coefficients $\Theta_{n}(t)$ in Eq. (50)

Having substituted Expressions (50) and (67) into Eq. (47), we rewrite the latter equation in the form:

$\sum_{n=0}^{\infty} \Theta_{n}^{\prime}(t) v_{n}(R)=D_{l} \sum_{n=0}^{\infty} \Theta_{n}(t) v_{n}^{\prime \prime}(R)-\epsilon^{\prime}(t) \sum_{n=0}^{\infty} Q_{n} v_{n}(R)$

where

$\Theta_{n}^{\prime}=\frac{\mathrm{d} \Theta_{n}}{\mathrm{~d} t} ; v_{n}^{\prime \prime}(R)=\frac{\mathrm{d}^{2} v_{n}}{\mathrm{~d} R^{2}} ; \epsilon^{\prime}(t)=\frac{\mathrm{d} \epsilon}{\mathrm{d} t} \equiv \epsilon^{\prime}$.

Since the expansion in the series with respect to $v_{n}$ (Fourier series) is unique, Eq. (70) is satisfied only when it is satisfied for each term in this expansion. Remembering that

$v_{0}^{\prime \prime}=\left(\frac{\lambda_{0}}{R_{d}}\right)^{2} v_{0}$ and $v_{n}^{\prime \prime}=-\left(\frac{\lambda_{n}}{R_{d}}\right)^{2} v_{n}(n \geq 1)$,

we can see that this implies that:

$\Theta_{0}^{\prime}(t)=D_{l}\left(\frac{\lambda_{0}}{R_{d}}\right)^{2} \Theta_{0}(t)-\epsilon^{\prime} Q_{0}$

$\Theta_{n}^{\prime}(t)=-D_{l}\left(\frac{\lambda_{n}}{R_{d}}\right)^{2} \Theta_{n}(t)-\epsilon^{\prime} Q_{n}$

when $n \geq 1$.

Eqs. (71) and (72) need to be solved subject to the initial conditions for $\Theta_{n}(t)(n \geq 0)$. To find these initial conditions we substitute (50) into the initial condition (49) and expand $R Y_{l i 0}(R)$ into a Fourier series with respect to $v_{n}$. Remembering that the expansion with respect to $v_{n}$ is unique, this leads us to the following equation for $\Theta_{n}(0)$ :

$\Theta_{n}(0)=q_{\text {in }}-\epsilon(0) Q_{n}$,

where $Q_{n}$ is defined by (68),

$q_{\text {in }}=\frac{1}{\left\|v_{n}\right\|^{2}} \int_{0}^{R_{d}} R Y_{l i 0}(R) v_{n}(R) \mathrm{d} R$

$n \geq 0$.

The solutions to Eqs. (71) and (72) subject to the initial condition (73) can be presented in the form:

$$
\begin{aligned}
\Theta_{0}(t)= & \exp \left[D_{l}\left(\frac{\lambda_{0}}{R_{d}}\right)^{2} t\right]\left[q_{i 0}-\epsilon(0) Q_{0}\right] \\
& -Q_{0} \int_{0}^{t} \frac{\mathrm{d} \epsilon(\tau)}{\mathrm{d} \tau} \exp \left[D_{l}\left(\frac{\lambda_{0}}{R_{d}}\right)^{2}(t-\tau)\right] \mathrm{d} \tau,
\end{aligned}
$$




$$
\begin{aligned}
\Theta_{n}(t)= & \exp \left[-D_{l}\left(\frac{\lambda_{n}}{R_{d}}\right)^{2} t\right]\left[q_{i n}-\epsilon(0) Q_{n}\right] \\
& -Q_{n} \int_{0}^{t} \frac{\mathrm{d} \epsilon(\tau)}{\mathrm{d} \tau} \exp \left[-D_{l}\left(\frac{\lambda_{n}}{R_{d}}\right)^{2}(t-\tau)\right] \mathrm{d} \tau,
\end{aligned}
$$

where $n \geq 1$.

\section{The final solution}

Having substituted (56), (61), (75) and (76), into (50), we can present the final solution to Eq. (15), satisfying boundary condition (16) and the corresponding initial condition in the form:

$$
\begin{aligned}
Y_{l i}= & \epsilon_{i}+\frac{1}{R}\left\{\left[\exp \left[D_{l}\left(\frac{\lambda_{0}}{R_{d}}\right)^{2} t\right]\left[q_{i 0}-\epsilon_{i}(0) Q_{0}\right]\right.\right. \\
& \left.-Q_{0} \int_{0}^{t} \frac{\mathrm{d} \epsilon_{i}(\tau)}{\mathrm{d} \tau} \exp \left[D_{l}\left(\frac{\lambda_{0}}{R_{d}}\right)^{2}(t-\tau)\right] \mathrm{d} \tau\right] \sinh \left(\lambda_{0} \frac{R}{R_{d}}\right) \\
& +\sum_{n=1}^{\infty}\left[\exp \left[-D_{l}\left(\frac{\lambda_{n}}{R_{d}}\right)^{2} t\right]\left[q_{i n}-\epsilon_{i}(0) Q_{n}\right]\right. \\
& \left.\left.-Q_{n} \int_{0}^{t} \frac{\mathrm{d} \epsilon_{i}(\tau)}{\mathrm{d} \tau} \exp \left[-D_{l}\left(\frac{\lambda_{n}}{R_{d}}\right)^{2}(t-\tau)\right] \mathrm{d} \tau\right] \sin \left(\lambda_{n} \frac{R}{R_{d}}\right)\right\},
\end{aligned}
$$

where $Q_{n}, q_{i n}, \lambda_{0}$ and $\lambda_{n}(n \geq 1)$ are defined by Eqs. (69), (74), (55) and (60) respectively; the subscript ${ }_{i}$ at $\epsilon$ has been restored.

Note that Expression (77) contains the term which exponentially increases with time. This, however, will not lead to an unphysical solution to Eq. (15), since this equation is valid only for 0 $\leq Y_{l i} \leq 1$. Once $Y_{l i}$ reaches one of its limiting values it will remain equal to this value.

If we apply Solution (77) to individual short timesteps, we can ignore the time dependence of $\mathrm{d}_{i}(\tau) / \mathrm{d} \tau$ during this time step and assume that

$\frac{\mathrm{d} \epsilon_{i}(t)}{\mathrm{d} t}=\left.\frac{\mathrm{d} \epsilon_{i}(t)}{\mathrm{d} t}\right|_{t=0} \equiv \epsilon_{i}^{\prime}$

This allows us to simplify Expression (77) to:

$$
\begin{aligned}
Y_{l i}= & \epsilon_{i}+\frac{1}{R}\left\{\left[\exp \left[D_{l}\left(\frac{\lambda_{0}}{R_{d}}\right)^{2} t\right]\left[q_{i 0}-Q_{0}\left(\epsilon_{i}(0)+\frac{R_{d}^{2}}{D_{l} \lambda_{0}^{2}} \epsilon_{i}^{\prime}\right)\right]\right.\right. \\
& \left.+Q_{0} \frac{R_{d}^{2}}{D_{l} \lambda_{0}^{2}} \epsilon_{i}^{\prime}\right] \sinh \left(\lambda_{0} \frac{R}{R_{d}}\right)+\sum_{n=1}^{\infty}\left[\exp \left[-D_{l}\left(\frac{\lambda_{n}}{R_{d}}\right)^{2} t\right]\right. \\
& \left.\left.\times\left[q_{i n}-Q_{n}\left(\epsilon_{i}(0)-\frac{R_{d}^{2}}{D_{l} \lambda_{n}^{2}} \epsilon_{i}^{\prime}\right)\right]-Q_{n} \frac{R_{d}^{2}}{D_{l} \lambda_{n}^{2}} \epsilon_{i}^{\prime}\right] \sin \left(\lambda_{n} \frac{R}{R_{d}}\right)\right\} .
\end{aligned}
$$

If we ignore the time dependence of $\epsilon_{i}$ then Expression (78) can be simplified to

$$
\begin{aligned}
Y_{l i}= & \epsilon_{i}+\frac{1}{R}\left\{\exp \left[D_{l}\left(\frac{\lambda_{0}}{R_{d}}\right)^{2} t\right]\left[q_{i 0}-\epsilon_{i}(0) Q_{0}\right] \sinh \left(\lambda_{0} \frac{R}{R_{d}}\right)\right. \\
& \left.+\sum_{n=1}^{\infty} \exp \left[-D_{l}\left(\frac{\lambda_{n}}{R_{d}}\right)^{2} t\right]\left[q_{i n}-\epsilon(0) Q_{n}\right] \sin \left(\lambda_{n} \frac{R}{R_{d}}\right)\right\} .
\end{aligned}
$$

The assumption that the time dependence of $\epsilon_{i}$ can be ignored during the time step will be used in our analysis. This allows us to base our calculations on Eq. (79).

\section{References}

[1] S.S. Sazhin, Advanced models of fuel droplet heating and evaporation, Prog. Energy Combust. Sci. 32 (2006) 162-214.

[2] M. Klingsporn, U. Renz, Vaporization of a binary unsteady spray at high temperature and high pressure, Int. J. Heat Mass Transfer 37 (Suppl. 1) (1994) $265-272$.

[3] P.L.C. Lage, C.M. Hackenberg, R.H. Rangel, Nonideal vaporization of dilating binary droplets with radiation absorption, Combust. Flame 101 (1995) 36-44.

[4] D.J. Torres, P.J. O'Rourke, A.A. Amsden, Efficient multi-component fuel algorithm, Combust. Theory Model. 7 (2003) 67-86.

[5] C. Maqua, G. Castanet, F. Lemoine, Bi-component droplets evaporation: temperature measurements and modelling, Fuel 87 (2008) 2932-2942.

[6] J. Tamim, W.L.H. Hallett, Continuous thermodynamics model for multicomponent vaporization, Chem. Eng. Sci. 50 (1995) 2933-2942.

[7] M. Burger, R. Schmehl, K. Prommersberger, O. Schäfer, R. Koch, S. Wittig, Droplet evaporation modelling by the distillation curve model: accounting for kerosene fuel and elevated pressures, Int. J. Heat Mass Transfer 46 (2003) 4403-4412.

[8] W.A. Sirignano, G. Wu, Multicomponent-liquid-fuel vaporization with complex configuration, Int. J. Heat Mass Transfer 51 (2008) 4759-4774.

[9] S.S. Sazhin, A. Elwardany, P.A. Krutitskii, G. Castanet, F. Lemoine, E.M. Sazhina, M.R. Heikal, A simplified model for bi-component droplet heating and evaporation, Int. J. Heat Mass Transfer 53 (2010) 4495-4505.

[10] N. Nikolopoulos, A. Theodorakakos, G. Bergeles, A numerical investigation of the evaporation process of a liquid droplet impinging onto a hot substrate, Int. J. Heat Mass Transfer 50 (2007) 303-319.

[11] G. Strotos, M. Gavaises, A. Theodorakakos, G. Bergeles, Numerical investigation on the evaporation of droplets depositing on heated surfaces at low Weber numbers, Int. J. Heat Mass Transfer 51 (2007) 1516-1529.

[12] G. Strotos, M. Gavaises, A. Theodorakakos, G. Bergeles, Numerical investigation of the cooling effectiveness of a droplet impinging on a heated surface, Int. J. Heat Mass Transfer 51 (2007) 4728-4742.

[13] S.S. Sazhin, P.A. Krutitskii, I.G. Gusev, M.R. Heikal, Transient heating of an evaporating droplet, Int. J. Heat Mass Transfer 53 (13-14) (2010) 2826-2836.

[14] S.S. Sazhin, P.A. Krutitskii, I.G. Gusev, M.R. Heikal, Transient heating of an evaporating droplet with presumed time evolution of its radius, Int. J. Heat Mass Transfer 54 (5-6) (2011) 1278-1288.

[15] H.S. Carslaw, J.C. Jaeger, Conduction of Heat in Solids. Clarendon Press, Oxford, 1986.

[16] E.M. Kartashov, Analytical Methods in Heat Transfer Theory in Solids. Vysshaya Shkola, Moscow, 2001, (in Russian).

[17] B. Abramzon, W.A. Sirignano, Droplet vaporization model for spray combustion calculations, Int. J. Heat Mass Transfer 32 (1989) 1605-1618.

[18] G. Continillo, W.A. Sirignano, Unsteady, spherically-symmetric flame propagation through multicomponent fuel spray clouds. in: G. Angelino, L. De Luca, W.A. Sirignano (Eds.), Modern Research Topics in Aerospace Propulsion. Springer-Verlag, New York, Berlin, 1991, pp. 173-198.

[19] P. Atkins, J. de Paula, Atkins' Physical Chemistry, seventh ed. Oxford University Press, 2002.

[20] V. Deprédurand, G. Castanet, F. Lemoine, Heat and mass transfer in evaporating droplets in interaction: influence of the fuel, Int. J. Heat Mass Transfer 53 (2010) 3495-3502.

[21] T. Kristyadi, V. Deprédurand, G. Castanet, L. Lemoine, S.S. Sazhin, A. Elwardany, E.M. Sazhina, M.R. Heikal, Monodisperse monocomponent fuel droplet heating and evaporation, Fuel 89 (2010) 3995-4001.

[22] S.S. Sazhin, W.A. Abdelghaffar, E.M. Sazhina, M.R. Heikal, Models for droplet transient heating: effects on droplet evaporation, ignition, and break-up, Int. J. Thermal Sci. 44 (2005) 610-622.

[23] C. Maqua, G. Castanet, F. Grisch, F. Lemoine, T. Kristyadi, S.S. Sazhin, Monodisperse droplet heating and evaporation: experimental study and modelling, Int. J. Heat Mass Transfer 51 (2008) 3932-3945.

[24] S. Tonini, M. Gavaises, A. Theodorakakos, Modelling of high-pressure dense diesel sprays with adaptive local grid refinement, Int. J. Heat Fluid Flow 29 (2008) 427-448.

[25] S.S. Sazhin, V. Goldshtein, M.R. Heikal, A transient formulation of Newton's cooling law for spherical bodies, ASME J. Heat Transfer 123 (2001) 63-64.

[26] S.S. Sazhin, P.A. Krutitskii, S.B. Martynov, D. Mason, M.R. Heikal, E.M. Sazhina, Transient heating of a semitransparent spherical body, Int. J. Thermal Sci. 46 (2007) 444-457.

[27] S.S. Sazhin, I.G. Gusev, P.A. Krutitskii, M.R. Heikal, Transient heating of a semitransparent spherical body immersed into a gas with inhomogeneous temperature distribution. Int. J. Thermal Sci. 50 (2011) 1215-1228.

[28] P. Lavieille, F. Lemoine, G. Lavergne, G.,N. Lebouch, Evaporating and combusting droplet temperature measurements using two color laser induced fluorescence, Exp. Fluids 31 (2001) 45-55.

[29] C. Maqua, G. Castanet, N. Doué, G. Lavergne, F. Lemoine, Temperature measurements of binary droplets using three color laser-induced fluorescence, Exp. Fluids 40 (2006) 786-797.

[30] V. Deprédurand, P. Miron, A. Labergue, M. Wolff, G. Castanet, F. Lemoine, A temperature sensitive tracer suitable for two-colour laser-induced fluorescence thermometry applied to evaporating droplets, Meas. Sci. Technol. 19 (2008) $1-12$ 
[31] G. Castanet, A. Delconte, F. Lemoine, L. Mees, G. Grehan, Evaluation of temperature gradients within combusting droplets in linear stream using two colors laser-induced fluorescence, Exp. Fluids 39 (2005) 431-440.

[32] G. Knig, K. Anders, A. Frohn, A new light-scattering technique to measure the diameter of periodically generated moving droplets, J. Aerosol Sci. 17 (1986) 157-167.

[33] V. Deprédurand, Approche expérimentale de l' évaporation de sprays de combustibles multicomposant, Thesis of the Institut Polytechnique of Lorraine, France, 2009.

[34] S.S. Sazhin, W.A. Abdelghaffar, P.A. Krutitskii, E.M. Sazhina, M.R. Heikal, New approaches to numerical modelling of droplet transient heating and evaporation, Int. J. Heat Mass Transfer 48 (19-20) (2005) 4215-4228.
[35] B.E. Polling, J.M. Prausnitz, J.P. O’Connell, The Properties of Gases and Liquids. McGraw-Hill, 2000.

[36] L. Grunberg, A.H. Nissan, Mixture law for viscosity, Nature 164 (1949) 799-800.

[37] W.A. Sirignano, Fluid Dynamics and Transport of Droplets and Sprays. Cambridge University Press, Cambridge, 1999.

[38] S.S. Sazhin, T. Kristyadi, W.A. Abdelghaffar, M.R. Heikal, Models for fue droplet heating and evaporation: comparative analysis, Fuel 85 (2006) 1613-1630.

[39] S.S. Sazhin, P.A. Krutitskii, W.A. Abdelghaffar, S.V. Mikhalovsky, S.T. Meikle M.R. Heikal, Transient heating of diesel fuel droplets, Int. J. Heat Mass Transfer 47 (2004) 3327-3340. 